DOI 10.4171/JEMS/386

Gabriel Calsamiglia $\cdot$ Bertrand Deroin · Sidney Frankel · Adolfo Guillot

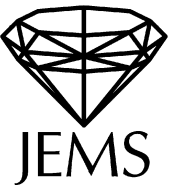

\title{
Singular sets of holonomy maps for algebraic foliations
}

Received November 30, 2010 and in revised form October 13, 2011

\begin{abstract}
In this article we investigate the natural domain of definition of a holonomy map associated to a singular holomorphic foliation of the complex projective plane. We prove that germs of holonomy between algebraic curves can have large sets of singularities for their analytic continuation. In the Riccati context we provide examples with natural boundary and maximal sets of singularities. In the generic case we provide examples having at least a Cantor set of singularities and even a nonempty open set of singularities. The examples provided are based on the presence of sufficiently rich contracting dynamics in the holonomy pseudogroup of the foliation. This gives answers to some questions and conjectures of Loray and Ilyashenko, which follow up on an approach to the associated ODE's developed by Painlevé.
\end{abstract}

\section{Introduction}

In the theory of real foliations on real manifolds, a standard approach to understanding the winding of the leaves of a foliation $\mathcal{F}$ within the ambient manifold is to consider a real (not necessarily connected or compact) submanifold $T$ (whose codimension is the dimension of the leaves) which intersects every leaf transversely, and to study the holonomy relation, the equivalence relation on $T$ whose classes are the intersections with each leaf. In this setting one has a refined construction whereby leafwise paths with endpoints in $T$ determine, by lifting to nearby leaves, a pseudogroup acting on $T$, called the holonomy pseudogroup, whose orbits are the holonomy classes. The study of this pseudogroup is central in foliation theory, insofar as it provides the dynamics of the foliation, as well as some useful geometric information.

In the complex context, in the setting of algebraic foliations of the complex projective plane, a very natural choice for $T$ is to consider an algebraic curve, with finite tangency

G. Calsamiglia: Instituto de Matemática, Universidade Federal Fluminense, Rua Mário Santos Braga s/n, 24020-140, Niterói, Brazil; e-mail: gabriel@mat.uff.br

B. Deroin: Département de Mathématiques d’Orsay, Université Paris 11, 91405 Orsay Cedex; e-mail: bertrand.deroin@math.u-psud.fr

S. Frankel: e-mail: sidney.frankel.mathsci@ gmail.com

A. Guillot: Instituto de Matemáticas, Unidad Cuernavaca, Universidad Nacional Autónoma de México, A.P. 273-3 Admon. 3, Cuernavaca, Morelos, 62251 Mexico; e-mail: adolfo@matcuer.unam.mx

Mathematics Subject Classification (2010): Primary 34M35; Secondary 32D15 
to $\mathcal{F}$. In fact, a generic curve $T$ intersects every leaf, as follows from the maximum principle of [6]. In this context, holonomy classes are no longer orbits of a pseudogroup of diffeomorphisms, due to the tangency of $T$ to the leaves. However, the situation is not so bad: the equivalence classes of the holonomy relation can still be described in terms of a set of local holomorphic correspondences on $T$, the algebroid class of Painlevé (see [30], [27], [10]).

In the more particular context of Riccatifoliations, holomorphic foliations in complex surfaces adapted to a rational fibration (in the sense that almost every fibre is everywhere transverse to the foliation), a natural choice for $T$ is one of the generic fibres. In this case, the holonomy classes are orbits of a finitely generated group of automorphisms of $\mathbb{P}^{1}$, known as the monodromy group, which governs the dynamics of the foliation.

We cannot hope, for a general algebraic foliation of the plane, that the holonomy relation can be realized as a genuine group of automorphisms of some Riemann surface transverse to the foliation, even after allowing for some (algebroid) ramified semi-conjugacy. Indeed, such a group necessarily preserves a projective structure on the surface (by uniformization), and it is well-known that the holonomy pseudogroups of generic plane algebraic foliations are not this rigid (see, for example, [3]).

In order to understand the nature and large scale behaviour of the holonomy correspondence between algebraic curves in a holomorphic foliation, one of the first problems is to determine the maximal domain where this correspondence is defined. Given a foliation and two curves $T_{1}$ and $T_{2}$ meeting a leaf transversely we obtain a germ of biholomorphism from $T_{1}$ to $T_{2}$ that is, a priori, analytic. It is thus natural to consider the problem of analytic extension of holonomy germs. The extension problem may be expressed in terms of determining the maximal domain of a holonomy correspondence. This domain is a curve, equipped with a projection into (not necessarily onto) $T_{1}$, where the analytic continuation of the germ is well-defined. Its boundary projects onto a subset of $T_{1}$, the set of singularities or singular set of the analytic continuation (see [27, Proposition 1.3] for details on this construction).

In this article, we investigate the set of singular points of the germ of a holonomy map, with domain and range lying within algebraic curves, and which is associated to a given singular algebraic foliation $\mathcal{F}$ of the complex projective plane $\mathbb{P}^{2}$. The nature of the set of singular points is tightly linked to other problems in the study of holomorphic foliations.

Firstly, it is possibly interesting, for the geometry and dynamics of foliations, to consider weaker analogues of product covering structures, with respect to an algebraic fibration $\mathbb{P}^{2} \rightarrow \mathbb{P}^{1}$. In other words, to find a correspondence analogous to "lifting", between leafwise paths, with good extension properties, and thereby view a foliation as if it were Riccati. The group of Möbius transformations would be replaced here by analytic continuation of holonomy germs (in which case, the dynamics of the foliation would be governed by the semigroup generated by the branches of a multivalued map, as studied in [7]).

In his presentation of Painlevé's Theorems (and in connection with them), Loray conjectured that the analytic continuation of such a germ is possible along any path in $T$ that avoids a countable set of points, the singularities [27, Conjecture 1]. Loray comments that, if this conjecture were true, the monodromy pseudogroup could be replaced by a 
very consistent monodromy group, and this would open the door to a Galois theory of such foliations.

In connection with the problem of simultaneous uniformization of the leaves of a foliation, Ilyashenko proved that some geometric objects in foliations (including holonomy maps) having a simultaneous uniformization have many good extension properties [18]. Any foliation where these extension properties fail thus exhibits an obstruction for the foliation to be simultaneously uniformizable. For example, the appearance of natural boundaries in the domain where the holonomy is defined is such an obstruction (see [18] for definitions and details). In this spirit, Ilyashenko asked [17, Problem 8] whether the analytic continuation of a given holonomy germ between rational curves is defined along most real rays (see also Problems 8.7 and 8.8 in [16]).

Our results show that, in the presence of rich contracting dynamics, there are special choices of the algebraic curve $T$ such that some holonomy correspondence has a maximal domain of definition whose boundary projects onto a large subset of $T$ (the set of singularities). We produce here examples of transversals in foliations where the analytic continuations of the holonomy germs have natural boundaries, where the singularities of these germs contain Cantor sets of positive dimension, and even open sets (Theorems 2 through 5). No examples with uncountably many singularities were previously known. On the opposite side of the spectrum, we prove that Loray's Conjecture holds in the absence of such rich dynamics (Theorem 1).

While our examples show that Loray's conjecture, as stated in [27], is false, the very interesting $\mathrm{PhD}$ thesis of Hussenot (see [15]) has appeared since our article was submitted. He proves that a modified version of Loray's conjecture in the context of Riccati foliations holds true: given a generic Riccati foliation of $\mathbb{P}^{1} \times \mathbb{P}^{1}$, and two generic algebraic curves $C, D \subset \mathbb{P}^{1} \times \mathbb{P}^{1}$, any holonomy germ from $C$ to $D$ extends along a.e. Brownian path in $C$. This leads to ask if Loray's conjecture could be correct in this weaker form, replacing "the paths that avoid a countable set of points in $C$ " by "a.e. Brownian path in $C$ ".

In order to state our results, let us recall some standard definitions. Let $\left(C_{0}, p_{0}\right)$ and $\left(C_{1}, p_{1}\right)$ be two pointed algebraic curves in $\mathbb{P}^{2}$ such that $p_{0}$ and $p_{1}$ belong to the same leaf $L$ of the holomorphic foliation $\mathcal{F}$. We suppose that for $i=0,1, p_{i}$ does not belong to the singular set, $\operatorname{Sing}(\mathcal{F})$, of $\mathcal{F}$, and that the curve $C_{i}$ is transverse to $\mathcal{F}$ at $p_{i}$. Consider a leafwise path $\gamma:[0,1] \rightarrow L$ such that $\gamma(0)=p_{0}$ and $\gamma(1)=p_{1}$. Then one can find a continuous family of leafwise paths $\gamma^{p}$, parametrized by a point $p \in C_{0}$ close enough to $p_{0}$, such that $\gamma^{p}(0)=p, \gamma^{p}(1)$ belongs to $C_{1}$, and $\gamma^{p_{0}}=\gamma$. The germ of the map $p \mapsto h_{\gamma}(p)=\gamma^{p}(1)$ is uniquely determined by the relative (i.e. with endpoints fixed) homotopy class of $\gamma$ under the above conditions, and is called the holonomy germ associated to $\gamma$. We will call such a germ between algebraic curves an admissible germ for $\mathcal{F}$.

Let $\left(C_{0}, p_{0}\right)$ and $\left(C_{1}, p_{1}\right)$ be two pointed complex curves and let $h:\left(C_{0}, p_{0}\right) \rightarrow$ $\left(C_{1}, p_{1}\right)$ be the germ of a holomorphic map. A point $q \in C_{0}$ is called a singularity of $h$ if there exists a path $\tau:[0,1] \rightarrow C_{0}$ such that $\tau(0)=p_{0}, \tau(1)=q$ and $h$ admits an analytic continuation along $\tau([0,1))$ by germs of holomorphic maps from $C_{0}$ to $C_{1}$ but not along $\tau([0,1])$ (see [27]). In all our examples, the singularities we will encounter are of a topological nature: $h$ will not even admit a continuous extension along $\tau([0,1])$. The 
set of singularities for $h$ could, in principle, be any subset of $C_{0}$. If it is the whole of $C_{0}$ we will say that $h$ has full singular set. There may also exist an open set $D \subset C_{0}$ containing $p_{0}$ such that for any path $\tau:[0,1] \rightarrow C_{0}$ starting at $p_{0}$ and such that $\tau(1) \in$ $\partial D, \tau^{-1}(D)=[0,1)$, the function $h$ has an analytic continuation along $\tau([0,1))$ but does not have an analytic continuation along $\tau([0,1])$. In the case where $\partial D$ is a topological circle, the analytic extension of $h$ is said to have $\partial D$ as its natural boundary.

Our first result shows that the phenomena discovered by Marín [29] in some particular foliations extend to all foliations given by closed meromorphic forms:

Theorem 1. Let $\mathcal{F}$ be a singular foliation given by a closed meromorphic 1-form on $\mathbb{P}^{2}$. Then the set of singularities of an admissible germ for $\mathcal{F}$ between rational curves is at most countable.

In other words, these foliations satisfy Loray's Conjecture. Loray conjectured that the same is true for any admissible germ associated to a holomorphic singular foliation of $\mathbb{P}^{2}$ [27]. The following result exhibits examples where the property fails:

Theorem 2. There exist holomorphic foliations of $\mathbb{P}^{2}$ :

(1) having an admissible germ between lines with a natural boundary;

(2) having an admissible germ between lines with full singular set.

Although (1) seems to be a quantitatively weaker result than (2), they are qualitatively different: the points in the natural boundary are singular for any path leading to them whereas this is certainly not the case for all singularities in (2). Examples of (1) will be defined by a Riccati equation with a Fuchsian monodromy group. The natural boundary appears as the limit set of the monodromy group, and it is topological in nature: it is not possible to extend the holonomy germ continuously to any of its points. The examples of (2) are defined by using Riccati equations with monodromy group dense in $\operatorname{Aut}\left(\mathbb{P}^{1}\right)$.

Our next result states that part of the phenomena displayed by the foliations in Theorem 2(1) are well behaved under small perturbations of the transversal curves where the germs are defined, and in particular permits constructing such an admissible germ (i.e. from a line to itself) with a large singular set:

Theorem 3. There exists a foliation $\mathcal{F}$ in $\mathbb{P}^{2}$ and an open subset of pairs of lines, $P \subset$ $\left(\mathbb{P}^{2}\right)^{*} \times\left(\mathbb{P}^{2}\right)^{*}$, intersecting the diagonal, such that for every $\left(L_{0}, L_{1}\right) \in P$ there is an admissible germ from $L_{0}$ to $L_{1}$ whose analytic extension has a curve of singularities (in particular, an uncountable set).

Being Riccati equations, the foliations in Theorems 2 and 3 are very special examples (they are special even within Riccati equations and are constructed from some particular projective structures on complex curves). By employing the underlying topological machinery of these examples, we are able to exhibit large singular sets for admissible germs in most algebraic foliations of the plane: we prove that for a generic foliation, it is always possible to find an admissible germ from an algebraic curve to itself, whose set of singularities for the analytic continuation is a large set, i.e. of positive dimension, and in particular uncountable. 
Theorem 4. Let $\mathcal{F}$ be a singular holomorphic foliation of $\mathbb{P}^{2}$ whose singularities are of hyperbolic type and with no invariant algebraic curve. Then there exists an admissible germ from a line to an algebraic curve whose singularity set for the analytic continuation contains a Cantor set.

It is also possible to construct an open set of foliations with admissible germs whose singular set has nonempty interior, thus extending the case of the foliation constructed in Theorem 2(2). Loray and Rebelo [28] exhibit, for every integer $d \geq 2$, a nonempty open set $\mathcal{U}_{d}$ in the parameter space of degree $d$ plane algebraic foliations such that every foliation belonging to $\mathcal{U}_{d}$ has dense leaves, and other strong ergodic properties.

Theorem 5. For every $d \geq 2$ and every singular holomorphic foliation belonging to a nonempty open subset of $\mathcal{U}_{d}$, there is an admissible germ from a line to an algebraic curve whose singularity set contains a nonempty open set.

We notice that the algebraic curve in Theorems 4 and 5 can be made rational. A small perturbation of the union of the line with the original algebraic curve would provide a nonsingular rational curve, and after a birational change of coordinates, an example of an admissible germ from a line to itself with the same conclusions. We will however not provide the details here. We finish by mentioning that Theorems 4 and 5 are also still true for small perturbations of the curves where the admissible germs are defined. The proof of this statement essentially follows the ideas used to prove Theorem 3 and will not be included in this work. Also, the same results most likely hold in the more general context of generic foliations on any complex algebraic surface, but we will not attempt to present things here at that level of generality.

The article is organized as follows. In Section 2 we discuss the case of foliations defined by closed meromorphic 1 -forms, and prove Theorem 1 . The method is quite different from that of [29] and uses arguments involving Painlevé's Theorem I. We also prove that, in some special cases, the analytic extension of a holonomy germ is still a holonomy germ. This section is essentially independent of what follows. Theorems 2 and 3 are proved in Section 3. Introductory material for the proofs is developed in Subsection 3.1, where it is proved that the inverse of the developing map of certain projective structures on curves has large sets of singularities. We also provide explicit polynomial forms for Theorems 2 and 3 in Subsection 3.4 and prove that the constructed foliations fail to admit a continuous bounded simultaneous uniformization in Subsection 3.6. In Section 4 we prove Theorems 4 and 5. This section can be read independently. Following the proofs, some problems left open are suggested in an afterword (Section 5), and some geometric discussion/explanation of the main ideas is added.

\section{Foliations defined by closed 1-forms}

In this section we exhibit a class of foliations for which admissible germs between rational curves possess small singular sets for their analytic continuation. In his thesis [29], Marín found explicit examples where Loray's Conjecture can be verified. In fact, his family 
can be extended to the family of all foliations defined by meromorphic closed 1-forms on $\mathbb{P}^{2}$. These admit a multivalued holomorphic first integral whose monodromy preserves a Riemannian metric, and which allows one to define, on each noninvariant algebraic curve $T$, a singular metric invariant under the holonomy pseudogroup. The problem of analytic continuation then reduces to the completeness of this metric. Since our argument only uses the codimension, we obtain:

Proposition 6. Let $\mathcal{F}$ be a codimension one holomorphic singular foliation defined by a meromorphic closed 1-form $\omega$ on a compact complex manifold $M$. Suppose that $L_{0}$ and $L_{1}$ are rational curves in $M$ and $h_{\gamma}:\left(L_{0}, p_{0}\right) \rightarrow\left(L_{1}, p_{1}\right)$ is an admissible germ for $\mathcal{F}$ associated to a leafwise path $\gamma$. Then the set of singularities of $h_{\gamma}$ is an at most countable set in $L_{0}$.

In particular, this is true for germs of holonomy associated to meromorphic fibrations $f: M \rightarrow \mathbb{C} P^{1}$. Theorem 1 is a direct consequence of Proposition 6. As we will see in Sections 3.2 and 4 the hypothesis $d \omega=0$ is crucial. Notice that it allows one to define a transversely Euclidean structure for the foliation on a Zariski open subset. A question that this leaves open is whether Proposition 6 extends to foliations that admit a transverse Riemannian structure on an invariant (Zariski) open set.

Proof of Proposition 6. Let $\omega_{i}(i=0,1)$ be the meromorphic 1-form on the rational curve $L_{i}$ defined by restriction of $\omega$ to $L_{i}$. We claim that the germs of 1 -forms $h_{\gamma}^{*}\left(\omega_{1}\right)$ and $\omega_{0}$ coincide at $p_{0}$. To prove the claim, let $U$ be the Zariski open set where $\omega$ is holomorphic. For any path $c$ in $U$ starting at $p_{0}=\gamma(0)$, the formula $F(c)=\int_{c} \omega$ defines a multivalued holomorphic first integral of $\mathcal{F}_{\left.\right|_{U}}$ on $U$ that forces the pole set $(\omega)_{\infty}$ to be invariant by $\mathcal{F}$. Hence, up to replacing $\gamma$ by its lift in a nearby leaf, we can suppose that $\gamma$ is contained in $U$. Let $q \in L_{0}$ be a point sufficiently close to $p_{0} \in L_{0}$. Consider a foliated homotopy $H:[0,1] \times[0,1] \rightarrow U$ such that for all $s, t \in[0,1]$ :

- $H(s, 0)=\gamma(s)$,

- $\tau_{0}(t):=H(0, t) \in L_{0}$,

- $\tau_{0}(1)=q$,

- $H(s, t)$ belongs to the leaf of $\mathcal{F}$ through $\tau_{0}(t)$,

- $\tau_{1}(t):=H(1, t)=h_{\gamma}\left(\tau_{0}(t)\right) \in L_{1}$.

Since $\omega$ is closed on $U$ an application of Stokes' Theorem shows that the integral of $\omega$ on the path described by going once around the boundary of the square defined by $H$ is zero. On the other hand for any path $c$ in a leaf of $\mathcal{F}$, we have $c^{*} \omega \equiv 0$. Hence

$$
\int_{\tau_{0}} \omega_{0}=\int_{\tau_{0}} \omega=\int_{\tau_{1}} \omega=\int_{h_{\gamma} \circ \tau_{0}} \omega_{1}=\int_{\tau_{0}} h_{\gamma}^{*}\left(\omega_{1}\right) .
$$

The equality implies that $h_{\gamma}^{*}\left(\omega_{1}\right)=\omega_{0}$ as germs at $p_{0}$. Since meromorphic 1-forms on rational curves are rational, we can write the 1-forms in coordinates to find rational functions $R, S$ such that $\omega_{0}(x)=R(x) d x$ and $\omega_{1}(y)=S(y) d y$. The germ $y=h_{\gamma}(x)$ is a solution to the differential equation

$$
\frac{d y}{d x}=\frac{R(x)}{S(y)} .
$$


Painlevé's Theorem I (see the Introduction and Deuxième Leçon in [30] and Loray's survey [27, Théorème (I), p. 14]) states that the solutions of rational differential equations admit analytic extensions along any path avoiding a countable set in the $x$ variable, i.e. in $L_{0}$. Hence the result.

\subsection{Admissible analytic extension of an admissible germ; the examples of Marín revisited}

In general it is not clear whether the analytic continuation of a holonomy germ is holonomic, and some care is needed even in the case of foliations defined by global rational functions. For instance consider the foliation $\mathcal{F}=\{d(x y)=0\}$ and the lines $L_{0}=\left\{y=y_{0}\right\}, L_{1}=\left\{x=x_{0}\right\}$, for fixed $x_{0}, y_{0} \neq 0$ close to 0 . The linear map $\left(x, y_{0}\right) \mapsto\left(x_{0}, x y_{0} / x_{0}\right)$ is a holonomy germ $\left(L_{0},\left(x_{1}, y_{0}\right)\right) \rightarrow L_{1}$ for $x_{1} \neq 0$ close to 0 but not for $x_{1}=0$.

Nevertheless, in his thesis, D. Marín [29] proved that for $\alpha \notin \mathbb{R}$ the foliation defined by $d y-y(y-1)(y-\alpha) d x=0$ in $\mathbb{P}^{1} \times \mathbb{P}^{1}$, which is Riccati with respect to $d y=0$, satisfies that any holonomy germ $h$ from a noninvariant fibre $F$ of $d x=0$ to itself can be analytically extended along any path avoiding a countable set in the fibre (we could use Proposition 6 here), and moreover the extension is still a holonomy map. He further proves that any two such germs of holonomy (different from the identity) can be obtained from each other by analytic continuation along paths in the fibre. We are going to adapt this example to the present setting and generalize it.

Proposition 7. Given a meromorphic 1 -form $\eta$ on $\mathbb{P}^{1}$ consider the foliation $\mathcal{F}$ defined by the meromorphic closed 1 -form $\omega$ on $\mathbb{P}^{1} \times \mathbb{P}^{1}$ whose expression in a chart is

$$
\omega(x, y)=d x-\eta(y), \quad(x, y) \in \mathbb{C}^{2},
$$

and an admissible germ $h$ for $\mathcal{F}$ between fibres of $\{d x=0\}$. If the analytic extension of $h$ along a path $\tau$ in the fibre that avoids $(\eta)_{\infty}$ is the germ of a biholomorphism at $\tau(1)$, then it is also an admissible germ for $\mathcal{F}$.

Proof. Let $\Sigma=\mathbb{P}^{1} \backslash\left\{p_{0}, \ldots, p_{d}\right\}$ be the set where $\eta$ is holomorphic. Denote by $P$ : $\widetilde{\Sigma} \rightarrow \Sigma$ a fixed universal covering map. By integration of $\eta$ along paths we can define a holomorphic mapping that corresponds to a branched projective structure (see definition in Section 3.1) $\mathcal{D}: \stackrel{\widetilde{\Sigma}}{\rightarrow} \mathbb{C}$ with critical points at the zero divisor $P^{-1}\left((\eta)_{0}\right)$ and with monodromy $\rho: \pi_{1}(\Sigma) \rightarrow \operatorname{PSL}(2, \mathbb{C})$. Furthermore its monodromy group $\Gamma=\operatorname{Im}(\rho)$ is a subgroup of translations of $\mathbb{C}$.

Notice that $(\omega)_{\infty}$ is invariant under $\mathcal{F}$ and contains the set $\operatorname{Sing}(\mathcal{F})$, so $\mathcal{F}$ is regular on $\mathbb{P}^{1} \times \mathbb{P}^{1} \backslash(\omega)_{\infty}=\mathbb{C} \times \Sigma$. The map (id, $P$ ) : $\mathbb{C} \times \widetilde{\Sigma} \rightarrow \mathbb{C} \times \Sigma$ is a universal covering map, and thanks to the closedness of $\omega$, the pullback of $\mathcal{F}$ is a regular foliation $\widetilde{\mathcal{F}}$ on $\mathbb{C} \times \widetilde{\Sigma}$ defined by the holomorphic 1 -form

$$
d(x-\mathcal{D}(y))=0 \quad \text { where } \quad(x, y) \in \mathbb{C} \times \widetilde{\Sigma} .
$$

Let $\gamma$ be a leafwise path such that $h_{\gamma}=h$ and $\tau$ as in the statement of the proposition. Lift them to paths in $\mathbb{C} \times \widetilde{\Sigma}$ with common basepoint and, by abuse of language, reuse the 
names $\gamma$ and $\tau$. We have $\gamma(0)=\left(x_{0}, y_{0}\right)$ and $\gamma(1)=\left(x_{1}, y_{1}\right)$ and suppose that both are transversality points of $\widetilde{\mathcal{F}}$ with the respective fibres of $d x=0$. The path $\tau$ is contained in the fibre $F_{0}=\left\{x_{0}\right\} \times \widetilde{\Sigma}$ and satisfies $\tau(0)=\gamma(0)$. Suppose $h_{\gamma}$ admits an analytic extension along $\tau$ (this is the case for most paths thanks to Proposition 6). We claim that the extended germs are still holonomy germs of $\widetilde{\mathcal{F}}$ at the points of $\tau$ where $F_{0}$ is transverse to $\widetilde{\mathcal{F}}$.

We have $\gamma(s)=\left(\gamma_{1}(s), \gamma_{2}(s)\right)$ and $\tau(t)=\left(x_{0}, \tau_{0}(t)\right)$. Let $c(t)=x_{0}-\mathcal{D}\left(\tau_{0}(t)\right) \in \mathbb{C}$. The path $h_{\gamma}(\tau(t))=:\left(x_{1}, \tau_{1}(t)\right) \in F_{1}$ satisfies $\tau_{1}(0)=\gamma_{2}(1)=y_{1}$ and $x_{1}-\mathcal{D}\left(\tau_{1}(t)\right)$ $=c(t)$ for small values of $t$, hence for all $t \in[0,1]$. Choose a homotopy $H:[0,1] \times[0,1]$ $\rightarrow \widetilde{\widetilde{\Sigma}}$ such that for each $(s, t) \in[0,1]^{2}$,

- $H(0, t)=\tau_{0}(t)$

- $H(1, t)=\tau_{1}(t)$

- $H(s, 0)=\gamma_{2}(s)$.

Then the map $F(s, t) \equiv(\mathcal{D}(H(s, t))+c(t), H(s, t)) \in \mathbb{C} \times \widetilde{\Sigma}$ defines for each $t \in[0,1]$ a path $\gamma_{t}$ in a leaf of $\widetilde{\mathcal{F}}$ with an endpoint at $\tau(t) \in F_{0}$ and the other at $h_{\gamma}(\tau(t)) \in F_{1}$. If neither of the endpoints belongs to $\operatorname{Tang}(\widetilde{\mathcal{F}}, d x=0)$, the holonomy germ associated to $\gamma_{t}$ realizes the analytic extension of the holonomy germ $h_{\gamma}$ along $\tau$. Finally we need to project the homotopy back to $\mathcal{F}$. It is enough to consider the composition (id, $P$ ) $\circ F$ : $[0,1]^{2} \rightarrow \mathbb{C} \times \Sigma$.

\section{Riccati foliations on the plane}

In this section we prove Theorems 2 and 3 . We begin by some introductory material concerning projective structures on curves.

\subsection{Projective maps between surfaces}

We will now introduce the main material which will serve to prove Theorems 2 and 3 . We consider branched projective structures on Riemann surfaces $\Sigma_{i}$ for $i=0,1$, and a germ of projective map (i.e. a composition of charts of the branched projective structures) between $\Sigma_{0}$ and $\Sigma_{1}$. We aim to provide examples of such germs whose analytic continuation has a large set of singularities. We begin with some classical facts on projective structures.

Let $\Sigma$ be a Riemann surface. A branched projective structure on $\Sigma=\bigcup U_{j}$ is a collection $\left\{\mathcal{D}_{j}\right\}$ of nonconstant holomorphic maps $\mathcal{D}_{j}: U_{j} \subset \Sigma \rightarrow \mathbb{P}^{1}$ such that the changes of coordinates (where defined) are Möbius transformations, i.e. $\mathcal{D}_{j} \circ \mathcal{D}_{k}^{-1}(z)$ has the form $(a z+b) /(c z+d)$. In fact any of the functions $\mathcal{D}_{j}$ can be extended analytically to a map $\mathcal{D}: \widetilde{\Sigma} \rightarrow \mathbb{P}^{1}$ defined on the universal cover $\widetilde{\Sigma}$ of $\Sigma$. These developing maps $\mathcal{D}$ are thus uniquely defined up to post-composition by a Möbius map, and they satisfy a formula of the form

$$
\mathcal{D}(\gamma(p))=\rho(\gamma) \mathcal{D}(p)
$$


for every $p \in \widetilde{\Sigma}$ and every $\gamma \in \pi_{1}(\Sigma)$, where $\rho: \pi_{1}(\Sigma) \rightarrow \operatorname{PSL}(2, \mathbb{C})$ is a certain representation associated to $\mathcal{D}$, called the monodromy representation. The monodromy representation, and hence its image, is well-defined up to conjugacy by a Möbius map, and we will refer to the conjugacy class of $\Gamma:=\rho\left(\pi_{1}(\Sigma)\right)$ in PSL $(2, \mathbb{C})$ as the monodromy group of the branched projective structure $\mathcal{D}$. The term branched comes from the fact that the maps $\mathcal{D}_{i}$ may have critical points. In fact the $\mathcal{D}_{j}$ define an orbifold structure on $\Sigma$ with projective changes of coordinates. If there are no critical points, they define a Riemann surface atlas with projective changes of coordinates and will be simply called a projective structure.

A projective structure on a punctured sphere $\Sigma=\mathbb{P}^{1} \backslash\left\{p_{1}, \ldots, p_{n}\right\}$ can be compared to the projective structure given by its natural embedding $\Sigma \hookrightarrow \mathbb{P}^{1}$ in the Riemann sphere, via the Schwarzian derivative defined for any function $f$ by $\{f, z\}=f^{\prime \prime \prime} / f^{\prime}-\frac{3}{2}\left(f^{\prime \prime} / f^{\prime}\right)^{2}$. More precisely, to any projective structure $\mathcal{D}$ on $\Sigma$ is canonically associated a quadratic form $q(z) d z^{2}$ by the formula

$$
q(z) d z^{2}:=\{\mathcal{D}, z\} d z^{2}
$$

which is independent of the chosen developing map. The quadratic form can also be defined in the case of a branched projective structure, but in this case it is meromorphic.

We will restrict our attention to parabolic type projective structures on $\Sigma$. Namely, in some coordinate $w$ around any puncture $p_{i}$, some developing map of our projective structure is given by

$$
\mathcal{D}=\frac{1}{2 \pi i} \log \left(w-w\left(p_{i}\right)\right) .
$$

This property is equivalent to the following Laurent series expansion of the quadratic differential $q(z) d z^{2}$ :

$$
\{\mathcal{D}, z\} d z^{2}=\left(\frac{1}{2\left(z-z\left(p_{i}\right)\right)^{2}}+\cdots\right) d z^{2}
$$

in the neighbourhood of any puncture $p_{i}$ (see [14, Chapter 10]). The set $\mathcal{P}$ of parabolic type projective structures on $\Sigma$ is a complex affine space directed by the vector space of meromorphic quadratic differentials on the sphere having at most simple poles at the punctures and being holomorphic in their complement. This space has positive dimension as soon as the number of punctures is at least 4 .

Examples of parabolic type projective structures on punctured spheres are given by uniformizations. For our constructions, we will need projective structures of parabolic type on punctured spheres with monodromy groups dense in $\operatorname{PSL}(2, \mathbb{C})$ :

Lemma 8. If $\Sigma$ is the sphere punctured by at least four points, then there exists a parabolic type projective structure on $\Sigma$ with dense monodromy.

Proof. Let us first assume that the monodromy of any parabolic type projective structure on $\Sigma$ is nonelementary, i.e. it is not conjugate to either a subgroup of the affine group, a subgroup of $\mathrm{SU}(2)$, or the group which preserves a nonoriented geodesic in $\mathbb{H}^{3}$. As we will see in Lemma 10 this is always the case. 
Let $\gamma \in \pi_{1}(\Sigma)$ and consider the map $T_{\gamma}: \mathcal{P} \rightarrow \mathbb{C}$ defined as $T_{\gamma}(\sigma)=\operatorname{Tr}^{2} \rho_{\sigma}(\gamma)$, where $\rho_{\sigma}$ is the monodromy of the projective structure $\sigma$. This map is holomorphic. We claim that there exists a nontrivial element $\gamma$ in $\pi_{1}(\Sigma)$ such that $T_{\gamma}$ is not constant. But if it were constant, a well-known fact is that the representation $\rho_{\sigma}$ would be constant up to conjugation (see [8, Proposition 1.5.2, p. 120]), equal to some representation $\rho$. Consider the $\mathbb{P}^{1}$-bundle constructed in Section 3.2 using the representation $\rho$, the compactification being given by the choice of integers $n_{p}=0$ for all cusps $p$ of $\Sigma$. Then the compactifications $\overline{\Delta_{\sigma}}$ of the diagonals defined by the projective structures $\sigma$ define a holomorphic family of different compact holomorphic curves, whose self-intersection is negative, by equation (3.3) in Section 3.2; this is impossible since holomorphic curves intersect nonnegatively. This proves the claim.

Then, because $\mathcal{P}$ is an affine complex space, $T_{\gamma}$ must take one of the values $2 \cos \alpha$ for an irrational real number $\alpha$, by Picard's Theorem. The monodromy $\rho_{\sigma}(\gamma)$ of the corresponding projective structure is conjugate to an irrational rotation, and thus the image of the monodromy representation is not discrete. Because the monodromy of a parabolic type projective structure is nonelementary, this implies that up to conjugation, either the monodromy is dense in $\operatorname{PSL}(2, \mathbb{R})$, or it is dense in $\operatorname{PSL}(2, \mathbb{C})$.

If it is dense in $\operatorname{PSL}(2, \mathbb{R})$, then by perturbing the projective structure, the image of the monodromy will still be nondiscrete. Indeed, by Zassenhaus's lemma [21, Theorem 4.52 , p. 85], a nonabelian subgroup generated by elements close enough to the identity is nondiscrete. Hence, by perturbing the projective structure so that $\operatorname{Tr}^{2}(\gamma)$ is not real, the image of $\rho_{\sigma}$ will be dense in $\operatorname{PSL}(2, \mathbb{C})$.

We will fix a hyperbolic Riemann surface $\Sigma$ with a branched projective structure, a developing map $\mathcal{D}$, and study the analytic continuation of the inverse $\mathcal{D}^{-1}$. We will prove that the singular set for the analytic continuation of this map is the limit set of the monodromy group, when either the projective structure is the uniformizing one, or the monodromy group is dense (in which case the limit set is the whole Riemann sphere).

Proposition 9. Let $\Sigma$ be a hyperbolic Riemann surface equipped with a branched projective structure and $\mathcal{D}$ be a developing map. Denote by $h$ a germ with extension the multivalued map $\mathcal{D}^{-1}$.

(1) If the projective structure is that given by uniformization, then h has natural boundary for its analytic continuation.

(2) If the monodromy group is dense in $\operatorname{PSL}(2, \mathbb{C})$, then $h$ has full singular set.

Before proceeding to the proof let us remark that we are going to find singularities of the inverse $h^{-1}$ based on an analysis of the asymptotic values of the germ $h$. Given a holomorphic germ $h:\left(\Sigma_{0}, p_{0}\right) \rightarrow\left(\Sigma_{1}, z_{0}\right)$ between complex curves, we say $z \in \Sigma_{1}$ is an asymptotic value for $h$ if there exists a path $\tau:[0,1) \rightarrow \Sigma_{0}$ such that $\tau(0)=p_{0}$, the analytic continuation of $h$ along $\tau$ exists and is a germ of biholomorphism at each point of $\tau$,

$$
z=\lim _{t \rightarrow 1} h(\tau(t)) \quad \text { but } \quad \lim _{t \rightarrow 1} \tau(t) \text { does not exist. }
$$

Hence $h^{-1}$ does not admit continuous extension to $z$ along $h \circ \tau$, which is enough for $z$ to be a singularity of $h^{-1}$. 
Proof of Proposition 9. (1) By the uniformization theorem for Riemann surfaces, $\Sigma$ is biholomorphic to the quotient of $\mathbb{D}$ by the action of a Fuchsian group preserving $\mathbb{D}$. The hypothesis states that, modulo conjugation by a Möbius map, $h^{-1}$ is a germ of the universal covering map $\mathbb{D} \rightarrow \Sigma$, hence it does not extend continuously to any point in the boundary of $\mathbb{D}$.

(2) Fix a point $p_{0}$ in $\widetilde{\Sigma}$ over $0 \in \Sigma$, and denote $z_{0}=\mathcal{D}\left(p_{0}\right)$. On the sphere $\mathbb{P}^{1}$, we consider the spherical metric of constant positive curvature given in affine coordinates $w$ by $|d s|=|d w| /\left(1+|w|^{2}\right)$. We denote by $D h$ the derivative of a Möbius map $h$ acting on the sphere, and by $|D h|(w)$ its spherical norm at the point $w$. If $w$ is a point of the sphere, we denote by $w^{\prime}$ the antipodal point.

For each point $z \in \mathbb{P}^{1}$ we will construct a finite set $\mathcal{A}$ in $\pi_{1}(\Sigma)$, and an infinite sequence $\alpha_{1}, \ldots, \alpha_{n}, \ldots$ of elements of $\mathcal{A}$, which have the following properties. Denoting $A_{n}:=\alpha_{1} \ldots \alpha_{n}$ (i.e. the group product), for every positive integer $n$, the diameter of the set (for the spherical metric)

$$
B_{n}:=\left\{w \in \mathbb{P}^{1}|| D\left(\rho\left(A_{n}\right)\right) \mid(w) \geq 1 / 2^{n}\right\}
$$

tends to 0 exponentially fast as $n \rightarrow \infty$, and the image $\rho\left(A_{n}\right)\left(\mathbb{P}^{1} \backslash B_{n}\right)$ is contained in $B\left(z, \mathrm{cst} / 2^{n}\right)$, where cst is a universal constant. Moreover, neither $z_{0}$ nor $\rho\left(\alpha_{n+1}\right)\left(z_{0}\right)$ belongs to $B_{n}$ (observe that this implies in particular that $\rho\left(A_{n}\right) z_{0}$ converges to $z$ as $n \rightarrow \infty)$.

Before proving the existence of such a sequence $\alpha_{n}$, let us explain why it implies the conclusion. The idea is to consider, for every nonnegative integer $n$, a smooth path $a_{n}:[0,1] \rightarrow \widetilde{\Sigma}$, which begins at $p_{0}$ and ends at $\alpha_{n}\left(p_{0}\right)$, of length bounded by a constant independent of $n$ (depending only on $\mathcal{A}$ ), and such that for every sufficiently large integer $n, \mathcal{D} \circ a_{n}$ does not take any value in the ball $B_{n-1}$. The reason why these paths $a_{n}$ exist is the following. Consider in $\widetilde{\Sigma}$ a large ball $D$ containing all the points $\alpha\left(p_{0}\right)$, where $\alpha$ ranges over $\mathcal{A}$. If $n$ is large enough, $B_{n-1}$ is a set in $\mathbb{P}^{1}$ with small diameter $\left|B_{n-1}\right|$, so that the set $D \cap \mathcal{D}^{-1}\left(B_{n-1}\right)$ is a disjoint union of topological balls of diameter bounded by cst $\cdot\left|B_{n-1}\right|$, and their number is bounded by the degree of $\mathcal{D}_{\mid D}$. To construct $a_{n}$, it suffices to follow the geodesic $\left[p_{0}, \alpha_{n}\left(p_{0}\right)\right]$ between $p_{0}$ and $\alpha_{n}\left(p_{0}\right)$ in $\widetilde{\Sigma}$, and to make a detour around the components of $\mathcal{D}^{-1}\left(B_{n-1}\right) \cap D$ each time the geodesic hits one of them.

We are ready to construct the path $c:[0, \infty) \rightarrow \widetilde{\Sigma}$. This is an infinite concatenation of the paths $c_{n}:=A_{n-1} a_{n}$, which starts at $A_{n-1}\left(p_{0}\right)$ and ends at $A_{n}\left(p_{0}\right)$; namely, we define $c(t)=c_{n}(t-n+1)$ for every nonnegative integer $n$ and every $t \in[n, n+1]$. Notice that $\mathcal{D} \circ c_{n}=\rho\left(A_{n-1}\right) \circ \mathcal{D} \circ a_{n}$, so that because $\mathcal{D} \circ a_{n}$ does not take any value in the ball $B_{n-1}$, the length of $\mathcal{D} \circ c_{n}$ goes to 0 exponentially fast. We deduce that $\mathcal{D} \circ c(t)$ converges to a point in $\mathbb{P}^{1}$ as $t \rightarrow \infty$; this point must be $z$ since $c(n)=\rho\left(A_{n}\right)\left(z_{0}\right)$ converges to $z$ as $n \rightarrow \infty$, and thus $z$ is an asymptotic value of $\mathcal{D}$.

It remains to prove the existence of the set $\mathcal{A}$ and of the sequence $\left(\alpha_{n}\right)$. Let $\delta>0$ be a small real number. We claim that it is possible to find a finite subset $\mathcal{B}$ of $\pi_{1}(\Sigma)$ such that, for every pair of points $\{u, v\}$ in $\mathbb{P}^{1}$ with $d\left(u, v^{\prime}\right) \leq \delta$, there is an element $\beta \in \mathcal{B}$ such that 
- $\rho(\beta)$ maps $u$ and $v$ to points $\delta / 2$-close to $z_{0}$ and $z_{0}^{\prime}$ respectively,

- $|D(\rho(\beta))|(u) \geq 4$ and $|D(\rho(\beta))|(v) \leq 1 / 4$.

Indeed, for every pair $\{u, v\}$ of points of the sphere such that $d\left(u, v^{\prime}\right) \leq \delta$, there is a Möbius map $h$ in $\operatorname{PSL}(2, \mathbb{C})$ such that $d\left(h(u), z_{0}\right)<\delta / 2, d\left(h(v), z_{0}^{\prime}\right)<\delta / 2$ and $|D h|(u)>4,|D h|(v)<1 / 4$. Because $\rho\left(\pi_{1}(\Sigma)\right)$ is dense in PSL $(2, \mathbb{C})$, we can suppose that the Möbius map $h$ belongs to the image of $\rho$. But then, the four preceding conditions will be satisfied if we move $u$ and $v$ a little. Thus, the claim is a consequence of the compactness of the set of pairs $\{u, v\}$ such that $d\left(u, v^{\prime}\right) \leq \delta$.

We construct a sequence of elements $\beta_{n}$ in $\mathcal{B}$ in the following way. The element $\beta_{1}$ is the element $\beta \in \mathcal{B}$ corresponding to the choice $u=z, v=z^{\prime}$. The element $\beta_{2}$ is the element of $\mathcal{B}$ corresponding to the choice $u=\beta_{1}(z)$ and $v=\beta_{1}\left(z^{\prime}\right)$, etc. By construction, $\beta_{n} \ldots \beta_{1}$ maps $z$ and $z^{\prime}$ to points $\delta / 2$-close to $z_{0}$ and $z_{0}^{\prime}$ respectively, and moreover

$$
\left|D\left(\beta_{n} \ldots \beta_{1}\right)\right|(z) \geq 4^{n} \quad \text { and }\left|D\left(\beta_{n} \ldots \beta_{1}\right)\right|\left(z^{\prime}\right) \leq 4^{-n} .
$$

Then we define $\mathcal{A}=\mathcal{B}^{-1}$ and $\alpha_{n}=\beta_{n}^{-1}$. We will prove that this sequence satisfies the required conditions.

We need to analyze the behaviour of the function $\left|D\left(\rho\left(A_{n}\right)\right)\right|$. Consider a Möbius map $R$ which sends $z$ and $z^{\prime}$ to $\rho\left(A_{n}\right)^{-1}(z)$ and $\rho\left(A_{n}\right)^{-1}\left(z^{\prime}\right)$ respectively, and which is 2bilipschitz. Such a map certainly exists since the points $\rho\left(A_{n}\right)^{-1}(z)$ and $\rho\left(A_{n}\right)^{-1}\left(z^{\prime}\right)$ are almost antipodal: more precisely, they are $\delta / 2$-close to $z_{0}$ and $z_{0}^{\prime}$, so we have $d\left(\rho\left(A_{n}\right)^{-1}(z)^{\prime}, \rho\left(A_{n}\right)^{-1}\left(z^{\prime}\right)\right) \leq \delta$. We will study the derivative of the Möbius map $\rho\left(A_{n}\right) \circ R$ instead of $\rho\left(A_{n}\right)$. Observe that $\left|D\left(\rho\left(A_{n}\right) \circ R\right)\right|=|D R| \cdot\left(\left|D\left(\rho\left(A_{n}\right)\right)\right| \circ R\right)$, and thus $\left|D\left(\rho\left(A_{n}\right)\right)\right|$ is the same as $\left|D\left(\rho\left(A_{n}\right) \circ R\right)\right| \circ R^{-1}$ up to a multiplicative constant. Changing affine coordinate if necessary without changing the expression of the spherical metric, we can suppose that $z^{\prime}=0$, hence $z=\infty$. Because $\rho\left(A_{n}\right) \circ R$ fixes $z$ and $z^{\prime}$, it is a homothety: $\rho\left(A_{n}\right) \circ R(w)=\lambda \cdot w$. A direct computation shows that

$$
\left|D\left(\rho\left(A_{n}\right) \circ R\right)\right|(w)=\frac{1+|w|^{2}}{|\lambda| \cdot|w|^{2}+|\lambda|^{-1}} .
$$

Observe that for every nonnegative integer $n$,

$$
|\lambda|=\left|D\left(\rho\left(A_{n}\right) \circ R\right)\right|(0) \geq \mathrm{cst} \cdot\left|D\left(\rho\left(A_{n}\right)\right)\right|\left(\rho\left(A_{n}\right)^{-1}\left(z^{\prime}\right)\right) \geq \mathrm{cst} \cdot 4^{n} .
$$

Thus, the derivative of $\rho\left(A_{n}\right) \circ R$ is larger than cst $\cdot 2^{-n}$ if and only if $|w| \leq \mathrm{cst} \cdot 2^{-n / 2}$. Hence, $B_{n} \subset R\left(\left\{|w| \leq \mathrm{cst} \cdot 2^{-n / 2}\right\}\right)$ and the diameter of $B_{n}$ tends exponentially fast to 0 .

It remains to prove that neither $z_{0}$ nor $\rho\left(\alpha_{n+1}\right)\left(z_{0}\right)$ belongs to $B_{n}$. We see that $\left.\mid D\left(\rho\left(A_{n}\right) \circ R\right)\right)\left.|(w) \leq \mathrm{cst} \cdot| \lambda\right|^{-1} \leq \mathrm{cst} \cdot 4^{-n}$ if $w \notin B\left(z^{\prime}, \delta\right)$. This implies that $\left|D\left(\rho\left(A_{n}\right)\right)\right|$ is bounded by cst $\cdot 4^{-n}$ outside the ball $R\left(B\left(z^{\prime}, \delta\right)\right) \subset B\left(z_{0}^{\prime}, 3 \delta\right)$. Because $z_{0} \notin B\left(z_{0}^{\prime}, 3\right)$, we have $\left|D A_{n}\right|\left(z_{0}\right) \leq \mathrm{cst} \cdot 4^{-n}$. Moreover,

$$
\left|D\left(\rho\left(A_{n}\right)\right)\right|\left(\rho\left(\alpha_{n+1}\right)\left(z_{0}\right)\right)=\frac{\left|D\left(\rho\left(A_{n+1}\right)\right)\right|\left(z_{0}\right)}{\left|D\left(\rho\left(\alpha_{n+1}\right)\right)\right|\left(z_{0}\right)} \leq \mathrm{cst} \cdot 4^{-n} .
$$

Hence, for $n$ sufficiently large, neither $z_{0}$ nor $\rho\left(\alpha_{n+1}\right)\left(z_{0}\right)$ belongs to $B_{n}$. Item (2) is proved. 


\subsection{Riccati foliation associated to a projective structure}

Given any parabolic type projective structure on a punctured sphere $\Sigma$, we proceed to the construction of some associated transversely projective foliations on rational complex surfaces. Fix a developing map $\mathcal{D}$ of the projective structure and the corresponding monodromy representation $\rho$. Consider the quotient of $\widetilde{\Sigma} \times \mathbb{P}^{1}$ by the action of $\pi_{1}(\Sigma)$ on it given by deck transformations on the first factor and $\rho$ on the second one. There are three holomorphic structures invariant under this action:

- the vertical fibration $\pi: \widetilde{\Sigma} \times \mathbb{P}^{1} \rightarrow \widetilde{\Sigma}$,

- the horizontal foliation $\mathcal{F}$, transverse to the fibration $\pi$,

- the section $\Delta:=\{(x, \mathcal{D}(x)): x \in \widetilde{\Sigma}\}$, which is transverse to both $\pi$ and the foliation at every point.

The quotient $S=\left(\widetilde{\Sigma} \times \mathbb{P}^{1}\right) / \pi_{1}(\Sigma)$ is a noncompact surface equipped with a $\mathbb{P}^{1}$-fibration $\pi: S \rightarrow \Sigma$, a smooth holomorphic foliation $\mathcal{F}$ transverse to $\pi$, and a holomorphic section $\Delta$ of $\pi$ transverse to $\mathcal{F}$ (for branched projective structures this has to be modified by considering tangencies between $\Delta$ and $\mathcal{F}$, but this does not affect the compactifications that follow). This construction is usually called the graph of the projective structure [12, Section 2].

Our goal is to compactify $(S, \pi, \mathcal{F}, \Delta$ ), where $\pi$ compactifies as a rational fibration over $\mathbb{P}^{1}$, namely a Hirzebruch surface (see [2]), but with a singular foliation. Above a neighbourhood of a cusp $p_{i}$, we have seen that the developing map $\mathcal{D}$ is given in some coordinates by $\mathcal{D}(w)=\frac{1}{2 \pi i} \log w$, where $w$ induces a biholomorphism from a neighbourhood $D_{i}$ of $p_{i}$ to the unit disc. Thus, the fibration $\pi$ restricted to $\pi^{-1}\left(D_{i}\right)$ is defined as the quotient of $\mathbb{H}^{2} \times \mathbb{P}^{1}$ by the action $(x, z) \mapsto(x+1, z+1)$, where $x:=\frac{1}{2 \pi i} \log w$, the foliation is the quotient of the horizontal foliation, and the curve $\Delta$ is the quotient of the diagonal $\{z=w\}$.

There exist local models of $\mathbb{P}^{1}$-bundles over a disc with a meromorphic flat connection with a pole at 0 , and parabolic monodromy (see [5, p. 53]): for any nonnegative integer $n$, they are defined by the differential equation

$$
w d t+\left(w^{n}-n t\right) d w=0
$$

in the coordinates $(w, t)$ belonging to $\mathbb{D} \times \mathbb{P}^{1}$. These foliations have either one or two singularities on the invariant fibre $w=0$, according to whether $n=0$ or $n>0$ respectively. In the $x$-coordinates, (3.1) reads $\frac{d t}{d x}=-e^{n x}+n t$ modulo $2 i \pi$, and the solutions are given by $t(x)=(\operatorname{cst}-x) \cdot e^{n x}$. Thus, the map $(x, z) \mapsto\left(x, t=(z-x) e^{n x}\right)$ induces a biholomorphism between $\pi^{-1}\left(D_{i}\right)$ and $\mathbb{D}^{*} \times \mathbb{P}^{1}$, sending the vertical fibration to itself, the foliation $\mathcal{F}$ to the foliation defined by (3.1), and the curve $\Delta$ to the curve whose equation is $\{t=0\}$.

Gluing the model (3.1) to the surface $S$ for each cusp of $\Sigma$, we obtain a compact complex surface $\bar{S}$. It is equipped with a rational fibration $\mathbb{P}^{1} \rightarrow \bar{S} \stackrel{\bar{\pi}}{\rightarrow} \mathbb{P}^{1}$, and a singular holomorphic foliation $\overline{\mathcal{F}}$ transverse to $\bar{\pi}$ except at the fibres over each cusp of $\Sigma$, where by the models (3.1) the fibre is invariant under the foliation $\overline{\mathcal{F}}$. Moreover, the section $\Delta$ compactifies to a section $\bar{\Delta}$ of $\bar{\pi}$ which passes through each singularity of $\overline{\mathcal{F}}$ of type (3.1) 
corresponding to a cusp $p$ with $n_{p}>0$, and such that $\bar{\Delta}$ is transverse to $\overline{\mathcal{F}}$ at each point of intersection, of a fibre over a cusp $p$ of $\Sigma$, iff $n_{p}=0$. We deduce (see [5, Proposition 2, p. 23]) that the number of tangencies counted with multiplicities is

$$
\operatorname{Tang}(\overline{\mathcal{F}}, \bar{\Delta})=\sum_{p} n_{p} .
$$

We claim that the self-intersection of $\bar{\Delta}$ is

$$
\bar{\Delta}^{2}=2+\sum_{p}\left(n_{p}-1\right)
$$

To prove this, observe that the normal bundle of $\bar{\Delta}$ is canonically isomorphic to the tangent bundle of the fibration $\bar{\pi}$ restricted to $\bar{\Delta}$, because $\bar{\Delta}$ is everywhere transverse to $\bar{\pi}$. Consider the morphism $P:\left.T \Delta \rightarrow T F\right|_{\Delta}$, from the tangent bundle of $\Delta \subset \bar{\Delta}$ to the tangent bundle of the fibration $F$, defined as the projection along the tangent bundle of $\mathcal{F}$. In the coordinates $(w, t)$ over a neighbourhood of the cusp $p$, the projection $P$ has the form

$$
P\left(\frac{\partial}{\partial w}\right)=w^{n_{p}-1} \frac{\partial}{\partial t} .
$$

This means that $P$ extends meromorphically from $T \bar{\Delta}$ to $\left.T F\right|_{\bar{\Delta}} \simeq N_{\bar{\Delta}}$, with a 0 of order $n_{p}-1$ at the cusp $p$, and we get the desired formula.

We recall here that by deforming the conformal structures of the punctured sphere, associated to base or fibre, we obtain foliations that are holomorphically different, and it has been remarked in [9, p. 821 after (1)] (see also [1, III.D, pp. 53-62], and note as well the beginning of our proof of Lemma 8) that only a very special subset of these admit holomorphic diagonals.

This provides some motivation to consider smooth real two-dimensional transversals, with the conformal structure they inherit from the foliation.

The previous construction allows us to prove the following lemma, which was used in the proof of Lemma 8.

Lemma 10. If $\Sigma$ is a sphere punctured at least at four points, then the monodromy of any parabolic type projective structure on $\Sigma$ is nonelementary.

Proof. Because the monodromy around the cusps is parabolic, it suffices to show that the monodromy is not conjugate to a subgroup of the affine group. But if it were, then the previously constructed foliation $\mathcal{F}$ associated to the parabolic type projective structure would have a leaf that gives a section of the fibration $S \rightarrow \Sigma$ (the leaf through a fixed point of the monodromy in $\mathbb{P}^{1}$, the point at infinity for the affine action). Taking the compactification corresponding to the integers $n_{p}=0$, one shows by a computation that this leaf would compactify as an invariant algebraic curve passing through each saddlenode singularity of $\overline{\mathcal{F}}$. By the Camacho-Sad formula [5, Theorem 2, p. 37], the selfintersection of such an invariant curve vanishes. This would imply that the $\mathbb{P}^{1}$-bundle $\bar{S}$ would be biholomorphic to the product $\mathbb{P}^{1} \times \mathbb{P}^{1}$, and would contradict the fact that there is a holomorphic section, $\bar{\Delta}$, of negative self-intersection. 


\subsection{Proof of Theorem 2}

Choose $d \in\{2,3, \ldots\}$. Consider the Riemann sphere punctured at $d+1$ points, together with its parabolic type uniformizing projective structure: take a curvilinear $(d+1)$-sided polygon in the unit disc $\mathbb{D} \subset \mathbb{P}^{1}$ with vertices in the unit circle $\mathbb{S}^{1}$ and consecutive sides $C_{i}, i=0, \ldots, d$, that are subarcs of circular arcs perpendicular to $\mathbb{S}^{1}$ at the vertices. Let $r_{i}$ be the hyperbolic reflection with respect to the circle containing $C_{i}$ and let $G$ denote the (Fuchsian) group of Möbius transformations whose generators are the compositions $\rho_{i}=$ $r_{i} \circ r_{i+1}$ for $i=0, \ldots, d$ where $r_{d+1}:=r_{0}$. By Poincaré's theorem (see for instance [32, Théorème VI.1.10, p. 211]), $G$ leaves $\mathbb{D}$ invariant and acts freely discontinuously there. The quotient map $\mathbb{D} \rightarrow \mathbb{D} / G=: \Sigma$ is a universal covering map of the curve $\Sigma$, which is biholomorphic to a $(d+1)$-punctured sphere, and we consider the projective structure given by the canonical equivariant embedding $\mathcal{D}: \widetilde{\Sigma}=\mathbb{D} \hookrightarrow \mathbb{P}^{1}$. By construction $\mathcal{D}$ is a parabolic type projective structure and by Proposition 9 a germ of its inverse $\mathcal{D}^{-1}$ has the unit circle $\mathbb{S}^{1}$ as natural boundary.

For the proof of item (2) consider a parabolic type projective structure $\mathcal{D}$ on a $d+2$ punctured sphere $\Sigma$ with dense monodromy, which exists by Lemma 8. Proposition 9 guarantees that a germ of the inverse $\mathcal{D}^{-1}$ has full singular set.

Construct the holomorphic foliation $\overline{\mathcal{F}}$ associated to the pair $(\Sigma, \mathcal{D})$, using the method at the beginning of this section, by choosing one $p_{0}$, setting $n_{p_{0}}=0$ and for all other $p$, setting $n_{p}=1$. Hence the compactification $\bar{\Delta}$ of the diagonal section $\Delta$ associated to $\mathcal{D}$ is a smooth rational curve of self-intersection +1 . This implies that the rational surface is the first Hirzebruch surface $\mathbb{F}_{1}$, which has a unique exceptional curve disjoint from $\bar{\Delta}$. Blowing down gives $\mathbb{P}^{2}$ equipped with a holomorphic foliation $\mathcal{G}$ of degree $d$ in the case of a Fuchsian representation, and of degree $d+1$ in the case of dense representation (by (3.2)). By construction there is an admissible germ for $\mathcal{G}$ from a fibre of the rational fibration (a line in $\mathbb{P}^{2}$ ) to the image of $\bar{\Delta}$ (another line in $\mathbb{P}^{2}$ ) that corresponds to the germ $\mathcal{D}^{-1}$ of $\overline{\mathcal{F}}$ and having the corresponding set of singularities as desired, because the restriction of the blowdown to a fibre (resp. $\bar{\Delta}$ ) is biholomorphic onto its image.

By using a different technique we provide, in Section 3.4, explicit polynomial 1-forms for the foliations constructed here.

\subsection{Explicit expressions}

In some particular cases, we can obtain birational models for the previously constructed Riccati foliations. We claim that we can find all the previous phenomena within the family of degree four foliations of $\mathbb{P}^{2}$ given in an affine chart by the kernel of the form

$$
\left(y^{2}[2 \lambda x+1]-2 x^{3}+L\right) y d x+\left(x^{4}-2 x y^{2}[\lambda x-1]+y^{4}-L x\right) d y,
$$

for $L=\left(1+\lambda^{2}\right) y^{2} x^{2}-2 \lambda y^{4}$ and $\lambda \in \mathbb{C}$. Blowing up the origin we pass from $\mathbb{P}^{2}$ to the first Hirzebruch surface, which is a rational fibration with rational base. In the coordinate chart $x=t y$ of the blowup we obtain the foliation $\mathcal{F}$ given by

$$
\left[1+2 t\left(\lambda-t^{2}\right) y+\left(t^{2}+t^{2} \lambda^{2}-2 \lambda\right) y^{2}\right] d t-\left(t^{4}-1\right) d y .
$$


In order to understand the foliation we will consider the foliation $\mathcal{F}_{2}$ that (3.5) induces in the second Hirzebruch surface $\mathbb{F}_{2}$, by means of the chart $(t, y)=\left(T^{-1}, Y T^{2}\right)$. The rational fibration $\Pi: \mathbb{F}_{2} \rightarrow \mathbb{P}^{1}$ determined by $\Pi(y, t)=[t: 1]$ is transverse to $\mathcal{F}_{2}$ except at the four exceptional fibres $\left\{t^{4}=1\right\}$ : the foliation is a Riccati one. In other words, if we let $\Sigma=\mathbb{P}^{1} \backslash\{1,-1, i,-i\}$ and $\Omega=\Pi^{-1}(\Sigma)$, then $\left.\Pi\right|_{\Omega}: \Omega \rightarrow \Sigma$ is a rational fibration everywhere transverse to $\mathcal{F}_{2}$. There is a section $\Delta: t \mapsto(0, t)$ of the fibration $\Pi$ that can be compactified to $\bar{\Delta}=\{y=0\}$, which is everywhere transverse to the foliation. Since we are in a transversely projective foliation, $\Delta$ inherits a projective structure that can be compared to the canonical one:

Proposition 11. The Schwarzian derivative of the projective structure induced by $\mathcal{F}$ with respect to the canonical one in $\Delta$ is given by

$$
2 \frac{4 t^{2}+\lambda\left(t^{4}-1\right)}{\left(t^{4}-1\right)^{2}} d t^{2}
$$

Proof. Recall that if $H(t)=\left(\begin{array}{ll}a(t) & b(t) \\ c(t) & d(t)\end{array}\right)$ is a solution to the linear differential equation

$$
\frac{d}{d t} H(t)=\left(\begin{array}{rr}
\frac{1}{2} \alpha_{1} & \alpha_{0} \\
-\alpha_{2} & -\frac{1}{2} \alpha_{1}
\end{array}\right) H(t),
$$

the general solution to the Riccati equation

$$
\left[\alpha_{2}(t) y^{2}+\alpha_{1}(t) y+\alpha_{0}(t)\right] \frac{\partial}{\partial y}+\frac{\partial}{\partial t}
$$

is given by

$$
h(t)\left(y_{0}\right)=\frac{a(t) y_{0}+b(t)}{c(t) y_{0}+d(t)}
$$

This implies that the flow of the vector field (3.8) in time $s$ is $(y, t) \stackrel{s}{\rightarrow}$ $\left(h(t+s) h^{-1}(t)(y), t+s\right)$ and hence $(y, t) \stackrel{t_{0}-t}{\longrightarrow}\left(h\left(t_{0}\right) h^{-1}(t)(0), t_{0}\right)$. The holonomy from $\Delta$ to the fibre $t=t_{0}$ is in consequence, up to a Möbius transformation, given by $\phi(t)=h^{-1}(t)(0)=-b(t) / a(t)$. We will now calculate its Schwarzian derivative. By (3.7), we have

$$
\frac{\phi^{\prime \prime}}{\phi^{\prime}}=\frac{\alpha_{0}^{\prime}}{\alpha_{0}}-\alpha_{1}-2 \frac{c}{a} \alpha_{0} .
$$

Developing $\{\phi(s), s\}=\left(\phi^{\prime \prime} / \phi^{\prime}\right)^{\prime}-\frac{1}{2}\left(\phi^{\prime \prime} / \phi^{\prime}\right)^{2}$ using (3.7) and the above formula, we obtain

$$
\{\phi(t), t\} d t^{2}=\left[2 \alpha_{0} \alpha_{2}-\frac{1}{2} \alpha_{1}^{2}-\alpha_{1} \frac{\alpha_{0}^{\prime}}{\alpha_{0}}-\alpha_{1}^{\prime}-\frac{3}{2}\left(\frac{\alpha_{0}^{\prime}}{\alpha_{0}}\right)^{2}+\frac{\alpha_{0}^{\prime \prime}}{\alpha_{0}}\right] d t^{2} .
$$

This is well-defined even in the presence of multivaluedness for the flow of the vector field. For equation (3.5), this gives exactly (3.6). 
Notice that for every fourth root of unity $t_{0}$, the expression (3.6) is, at $\tau=t-t_{0}$, of the form $\left(\frac{1}{2} \tau^{-2}+\cdots\right) d \tau^{2}$, and this implies that, at each one of these four points in $\bar{\Delta} \backslash \Delta$, the monodromy is parabolic (the point at $t=\infty$ is a regular point of the projective structure).

The monodromy representation $\rho: \pi_{1}(\Delta) \rightarrow \operatorname{PSL}(2, \mathbb{C})$ of the projective structure is the holonomy of the Riccati equation. If $\gamma:[0,1] \rightarrow \Delta$ is a closed path, since the holonomy from $\Delta$ to a generic fibre $F$ is a developing map $\mathcal{D}$ of the projective structure and $\mathcal{D}(\gamma \cdot p)=\rho(\gamma) \mathcal{D}(p)$, the difference of the holonomies is exactly $\rho(\gamma)$.

We can pull back the fibration $\mathbb{P}^{1} \rightarrow \Omega \rightarrow \Sigma$ to $\widetilde{\Sigma}$ via $\pi: \widetilde{\Sigma} \rightarrow \Sigma$ and obtain in this way a fibration $\mathbb{P}^{1} \rightarrow \widetilde{\Omega} \rightarrow \widetilde{\Sigma}$ endowed with a foliation $\widetilde{\mathcal{F}}_{2}$ that is transverse to the fibration. Up to a biholomorphism we can suppose that $\widetilde{\Omega}=\widetilde{\Sigma} \times \mathbb{P}^{1}$, and that $\widetilde{\mathcal{F}}_{2}$ is the horizontal foliation in $\widetilde{\Sigma} \times \mathbb{P}^{1}$. We may recover $\Omega$ by acting diagonally by $\pi_{1}(\Sigma)$ on $\widetilde{\Sigma} \times \mathbb{P}^{1}$ by deck transformations in the first factor and by some representation $\mu: \pi_{1}(\Sigma) \rightarrow \operatorname{PSL}(2, \mathbb{C})$ in the second one. This last representation must be the monodromy. Furthermore, in $\widetilde{\Sigma} \times \mathbb{P}^{1}$ the section $\Delta$ becomes the graph of a function $f: \widetilde{\Sigma} \rightarrow \mathbb{P}^{1}$, but the projection onto the second coordinate must be the developing map. We conclude that the foliations considered here belong to the family of previously constructed foliations. They correspond to the choice of $n_{p}=0$ at each of the four punctures of $\Sigma$. The surface $\mathbb{F}_{2}$ appears as a consequence of formula (3.3).

Since the dimension of the space of parabolic type projective structures on a four punctured sphere is one, equation (3.6) is an affine parametrization of that space in the case $\Sigma=\mathbb{P}^{1} \backslash\{1,-1, i,-i\}$. For the projective structure corresponding to the (Fuchsian) uniformization, the quadratic differential must be invariant under automorphisms of $\Sigma$. Under the symmetry $t \mapsto$ it the quadratic differential (3.6) is the same except for a change of sign in $\lambda$, that is, only $\lambda=0$ may give a quadratic differential which is invariant under the automorphisms of $\Sigma$ and corresponds thus to the uniformization parameter. For other values of the parameter $\lambda$, we find quasi-Fuchsian representations (for sufficiently small $\lambda$ ) and dense representations, as was shown in Lemma 8.

\subsection{Proof of Theorem 3}

We will consider the foliation (3.4) with $\lambda=0$ and the lines $C_{p, \epsilon}$ given by $\{x-y p-\epsilon=0\}$ for $|p|<1 / 4$. After the blowup leading to the foliation (3.5), the strict transform of $C_{p, \epsilon}$ becomes the curve $y=\epsilon(t-p)^{-1}$. This curve does not intersect $\Delta$. Let $K \subset \bar{\Delta}$ be the annulus $1 / 2<|t|<2$. The complement of $K$ in $\bar{\Delta}$ is a compact set free of singularities of the projective structure (the four punctures are contained in $K$ ). If $\epsilon$ is small enough, $C_{p, \epsilon}$ is close to $\bar{\Delta}$ along $K$ and thus, since $\bar{\Delta}$ is transverse to $\mathcal{F}$, we have a holonomy map $f: K \rightarrow C_{p, \epsilon}$ that is a biholomorphism onto its image.

Let $q \in \mathbb{C}, q^{4} \neq 1$. We have shown that there exists an open disc $D \subset\{t=q\}$ and a holonomy map $h: D \rightarrow \Delta$ that realizes the Fuchsian uniformization of $\Delta$ and that has $\partial D$ as natural boundary. Notice that, for every $z \in \partial D$ we can find a path $\gamma:[0,1] \rightarrow\{t=q\}$ such that $\gamma(1)=z$ and $\gamma(t) \in h^{-1}(K)$ for $t<1$. Consider the restriction $\left.h\right|_{h^{-1}(K)}: h^{-1}(K) \rightarrow K$. It is still a holonomy map and, through $\gamma$, has at $z$ a singularity for its analytic continuation. Hence, for every $z \in \partial D,\left.f \circ h\right|_{h^{-1}(K)}$ is a holonomy map from $\{t=q\}$ to $C_{p, \epsilon}$ for which $z$ is a singularity for its analytic con- 


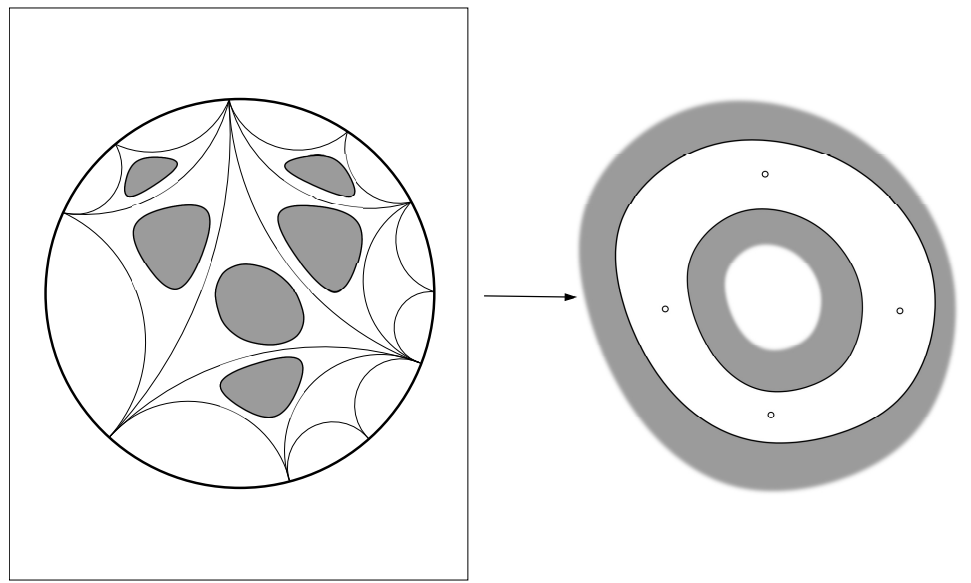

Fig. 1. Singularities of the holonomy in the perturbed line: through the holonomy relation, the complement of the shaded part within the circle realizes the universal covering of the annulus. This covering cannot be extended to the circle.

tinuation (see Figure 1). Notice that, because $\mathcal{F}$ is both transverse to $\Delta$ and to $\{t=q\}$ at the intersection of these curves, $\Delta$ intersects $\{t=q\}$ in the interior of $D$. Let $B \subset D$ be a small closed ball containing this point of intersection. If $\epsilon^{\prime}$ is small enough, $\mathcal{F}$ establishes a holonomy diffeomorphism $g:(\{t=q\} \backslash B) \rightarrow C_{q, \epsilon^{\prime}}$ defined, in particular, in $\partial D$. We have the holonomy $\left.f \circ h\right|_{h^{-1}(K)} \circ g^{-1}$ between $C_{q, \epsilon^{\prime}}$ and $C_{p, \epsilon}$. The holonomy is defined along $g \circ \gamma(t)$ for every $t<1$ but cannot be extended to $t=1$. In other words, the points of the curve $g(\partial D)$ are singularities for the analytic continuation of an admissible germ from $C_{q, \epsilon^{\prime}}$ to $C_{p, \epsilon}$. This ends the proof of Theorem 3.

It is worth noticing that if $\epsilon$ is small enough, the projective structure in $C_{p, \epsilon}$ can be completely understood: it is a bubbling (see [11]) over the projective structure of $\Delta$ along two arcs, one of them close to $\{t=p\}$ and the other close to $\{t=\infty\}$. This is, however, unnecessary for the proof of the theorem.

\subsection{Aside: Simultaneous uniformization}

A holomorphic foliation $\mathcal{F}$ on a surface $S$ with a given transversal $\Delta$ admits a simultaneous uniformization if the union $M=\bigcup_{q \in \Delta} \widetilde{L}_{q}$ of the universal covering spaces of the leaves intersecting $\Delta$ (with the analytic structure induced by the one in the leaves and the one in $\Delta$ ) admits a holomorphic embedding $\Phi: M \rightarrow \Delta \times \mathbb{P}^{1}$ such that, for every $q \in \Delta$, $\Phi(q)=(q, 0)$ and for every leaf $L$ the projection of $\Phi(L)$ onto $\Delta$ is constant (this notion was introduced in [20]). The simultaneous uniformization is continuous if $\Phi\left(L_{q}\right)$ varies continuously with $q$, and bounded if there exist $r, R \in \mathbb{R}$ such that $\Phi\left(L_{q}\right)$ contains the disc of radius $r$ and is contained in the disc of radius $R$. In [18], Ilyashenko proved that if a foliation $\mathcal{F}$ admits a continuous simultaneous bounded uniformization with respect 
to the tranversals $\Delta_{1}$ and $\Delta_{2}$, the holonomy map from $\Delta_{1}$ to $\Delta_{2}$ does not have a natural boundary.

By Ilyashenko's Theorem, the foliations related to the first part of Theorem 2 (where there is a natural boundary for the holonomy map) do not admit a continuous bounded simultaneous uniformization. Since, at first sight, it may seem striking that some Riccati foliations fail to exhibit good uniformization properties (boundedness in this case), we decided to include a direct proof of the absence of a continuous bounded uniformization for the foliations constructed for Theorem 2 (the ones corresponding to the projective structure associated to the uniformization).

Our setting is the following one: Let $\mathbb{D}$ be the unit disc in $\mathbb{C}, \Sigma$ an $n$-punctured sphere $(n \geq 3), \Pi: \mathbb{D} \rightarrow \Sigma$ the uniformization. Let $\mathcal{F}$ be the foliation in $\mathbb{D} \times \mathbb{D}$ given by the fibres of the projection onto the first factor and let $\Delta \subset \mathbb{D} \times \mathbb{D}$ be the diagonal. Let $S$ be the surface obtained by the quotient of $\mathbb{D} \times \mathbb{D}$ by the diagonal action of $\pi_{1}(\Sigma), \mathcal{F}$ and $\Delta$ the corresponding foliation and transversal in $S$.

Proposition 12. With respect to the transversal $\Delta$, the above constructed foliation $\mathcal{F}$ in $S$ admits a continuous simultaneous uniformization but does not admit a bounded one.

Proof. The covering $M=\bigcup_{q \in \Delta} L_{q}$ identifies to $S$, since every leaf of $\mathcal{F}$ is simply connected and intersects $\Delta$ once and only once. We have a projection $\Pi: M \rightarrow \Delta$ and a section $\sigma: \Delta \rightarrow M$. They are given, in $\mathbb{D} \times \mathbb{D}$, by the projection onto the first factor and by the embedding of the diagonal (before quotient).

We claim that there is no other section of $\Pi$. In fact, any section comes from a map $i: \mathbb{D} \rightarrow \mathbb{D} \times \mathbb{D}$ of the form $z \mapsto(z, f(z))$ that must be preserved by the diagonal action of $\pi_{1}(\Sigma)$, that is, for every $g \in \pi_{1}(\Sigma),(g(z), g(f(z)))=(g(z), f(g(z)))$, that is, $f \circ g=g \circ f$ (in particular, $f$ is not constant). The mapping $f$ induces a mapping $f^{b}: \Sigma \rightarrow \Sigma$. We will consider $\Sigma$ with its natural hyperbolic metric, where the punctures are cusps of infinite length and have neighbourhoods of arbitrarily small area. By the Schwarz-Pick Lemma ([22, Theorem 1.2]), $f^{b}$ is (hyperbolic) distance-decreasing and (hyperbolic) area-contracting. If we think of $\Sigma$ as embedded in $\mathbb{P}^{1}$ then, at the punctures of $\Sigma, f^{b}$ cannot have an essential singularity for, in such a case, by Picard's Big Theorem, a neighbourhood of a puncture of arbitrarily small hyperbolic area would be mapped to a set whose hyperbolic area is (at least) that of $\Sigma$. Hence, $f^{b}$ is meromorphic at the punctures and is thus the restriction of a rational function $R: \mathbb{P}^{1} \rightarrow \mathbb{P}^{1}$. Under $R$, the preimage of any puncture of $\Sigma$ is a puncture of $\Sigma$ for otherwise the distance-contracting property would be violated. Hence, $R$ maps the punctures of $\Sigma$ to the punctures of $\Sigma$. The rational function $R$ must have degree one by the area-contracting property of $f^{b}$ and is thus a Möbius transformation. Hence, $f: \mathbb{D} \rightarrow \mathbb{D}$ is a biholomorphism (given in fact by a Möbius transformation of $\mathbb{P}^{1}$ preserving $\partial \mathbb{D}$ ) that commutes with every element of $\pi_{1}(\Sigma)$. If $g \in \pi_{1}(\Sigma)$ is hyperbolic, it has two fixed points in $\partial \mathbb{D}$ that must also be fixed points of $f$. Since $\pi_{1}(\Sigma)$ has at least two hyperbolic elements with different fixed points, $f(z)=z$ and the section given by $i$ is $\Delta$.

Suppose now that we have a continuous uniformization $\Phi: M \rightarrow \Delta \times \mathbb{P}^{1}$ that is bounded from below, that is, that there exists some $r \in \mathbb{R}$ such that a disc of radius $r$ is in the image of $L_{q}$ for every $q \in \Delta$. This implies that $z \mapsto(z, r / 2)$ is a section of $S$ (after 
embedding it in $\Delta \times \mathbb{P}^{1}$ ). This contradiction proves that there is no continuous bounded simultaneous uniformization.

We will now show that the compactification constructed in Section 3.2 actually gives a continuous uniformization. We embedded the quotient of $\mathbb{D} \times \mathbb{D}$ (through its embedding into $\mathbb{D} \times \mathbb{P}^{1}$ ) into a Hirzebruch surface, by adding some lines. We may take a chart $\mathbb{C} \times \mathbb{P}^{1}$ within the complement of one of these lines in the Hirzebruch surface and, within this chart, after removing the other $\mathbb{P}^{1}$-fibres that were added, we find $\Sigma \times \mathbb{P}^{1}$, where we might embed $S$. One of the foliations in $S$ (they are both perfectly equivalent) is now tangent to the $\mathbb{P}^{1}$-fibres: we have a continuous simultaneous uniformization. In each fibre the boundary of $\mathbb{D}$ is a circle, which approaches the singularities of the foliation as we move away from $\Sigma$ and into the punctures, and in this way we can see how this particular simultaneous uniformization fails to be bounded (see [13, § 4.3] for more details on the structure of these foliations near these points).

\section{Generic foliations}

\subsection{Proof of Theorem 4}

The holonomy map with natural boundary (Theorem 2) was constructed using the quotient map of the domain of discontinuity of a Fuchsian group. Analogous maps, closely related to Schottky groups, can in fact be seen as the holonomy of generic foliations of the complex projective plane. The existence of rich dynamics in the holonomy pseudogroup will underlie their construction.

Before proceeding to an explanation, we need to introduce the concept of $\mathcal{F}$-homotopy, and to discuss a subtlety in the definition of the holonomy map. Given a compact topological space $X$, an algebraic foliation $\mathcal{F}$ of $\mathbb{P}^{2}$, and continuous maps $F_{0}, F_{1}$ : $X \rightarrow \mathbb{P}^{2}$, an $\mathcal{F}$-homotopy between $F_{0}$ and $F_{1}$ is a continuous family of leafwise paths $\Gamma^{x}:[0,1] \rightarrow \mathbb{P}^{2}$ such that $\Gamma^{x}(i)=F_{i}(x)$ for $i=0,1$ and every $x \in X$. Sometimes the $\mathcal{F}$-homotopy will be denoted by $\Gamma(s, x)=\Gamma^{x}(s)$. Now, define a holonomy tube between two open sets $U, V$ contained in algebraic curves to be an $\mathcal{F}$-homotopy between $\operatorname{id}_{\bar{U}}$ and some map $h: \bar{U} \rightarrow \bar{V}$. Of course, the map $h$ associated to a holonomy tube is a holonomy map, but strictly speaking tubes have more structure, the reason being that in a holonomy map the homotopy class of the leafwise path is not uniquely determined (see [33] for more details on the consequences of this nonuniqueness).

Lemma 13. Under the hypotheses of Theorem 4 there exists a line $L$, an open disc $B \subset L$ and a pair of holonomy tubes between $\bar{B}$ and the pair of subsets, $h_{i}(\bar{B}) \subset B$, where the associated holonomy maps $h_{1}, h_{2}: \bar{B} \rightarrow B$ are injective and such that

$$
h_{1}(\bar{B}) \cap h_{2}(\bar{B})=\emptyset .
$$

Proof. Let $T$ be an algebraic curve such that $\operatorname{Sing}(\mathcal{F}) \cap T=\emptyset$, and let $\mathcal{M}$ be a closed union of leaves of $\mathcal{F}$ such that $\mathcal{M} \cap T$ is minimal, for the holonomy relation on $T$ defined in the Introduction, over all such closed subsets. Note that $\mathcal{M}$ may accumulate on $p \in$ $\operatorname{Sing}(\mathcal{F})$. Furthermore, by an application of the maximum principle of [6], these are the 
only possible nontrivial $\mathcal{F}$-invariant subsets. Thus $\mathcal{M}$ is a quasi-minimal set for $\mathcal{F}$, that is, a nonempty closed invariant subset of $\mathbb{P}^{2} \backslash \operatorname{Sing}(\mathcal{F})$ such that every leaf $\mathcal{F}_{x} \subset \mathcal{F}$ is dense in $\mathcal{M}$.

We will now produce a closed leafwise path $\gamma:[0,1] \rightarrow \mathcal{F}_{x} \rightarrow \mathcal{M}$, based at some point $x \in \mathcal{M}$, and an open disc $B$ in a line of $\mathbb{P}^{2}$ containing $x$ and transverse to $\mathcal{F}$ such that the holonomy map $h_{\gamma}$ is defined on $B$ and satisfies $h_{\gamma}(\bar{B}) \Subset B$.

Suppose that $\overline{\mathcal{M}}$ contains a singularity $p$ of $\mathcal{F}$. By hypothesis $p$ is hyperbolic, so restricting to the domain of linearization of $\mathcal{F}$ at $p$, a leaf of $\mathcal{M}$ accumulates in a separatrix $S$ of $p$, so $S \subset \mathcal{M}$. There is then a loop $\gamma \subset S$ and a transverse disc $B$ producing the hyperbolic holonomy germ $\overline{h_{\gamma}(B)} \Subset B$ as desired. On the other hand, if $\overline{\mathcal{M}}$ does not contain a singularity of $\mathcal{F}$, then $\mathcal{M}$ is an exceptional minimal set and the existence of the germ $h_{\gamma}$ was proven in [4]. In any case $h_{\gamma}$ has a fixed point 0 (in a coordinate $z: B \rightarrow \mathbb{D}$ ) and by the Schwarz Lemma $h_{\gamma}^{n}(B) \rightarrow 0$ uniformly in $n$. Shrinking $B$ if necessary, we can construct a holonomy tube from $\bar{B}$ to $h_{\gamma}(\bar{B})$, which we denote by $\Gamma^{\prime}:[0,1] \times B \rightarrow \mathbb{P}^{2}$.

Since $\mathcal{F}$ has no invariant algebraic curves, $\mathcal{M}$ is transversely a perfect set. We can choose open sets (by increasing $n$ as needed) $U, V \subset B$ such that $z=0 \in U \Subset \bar{U} \Subset$ $h(B), \bar{V} \Subset B \backslash \overline{h(B)}$, and there exists a holonomy diffeomorphism $\psi: U \rightarrow V$ with $\psi(0) \in V$. Shrinking $U$ if necessary, we consider a holonomy tube $\Gamma^{\prime \prime}:[0,1] \times U \rightarrow \mathbb{P}^{2}$ from $U$ to $V$ whose associated map is $\psi$. Now for sufficiently large $n \in \mathbb{N}, h_{\gamma}^{n}(B) \Subset$ $U \cap \psi^{-1}(V)$, so by defining $h_{1}=h_{\gamma}$ and $h_{2}=\psi \circ h_{\gamma}^{n}$, we get two injective holonomy maps $h_{1}, h_{2}: \bar{B} \rightarrow B$ of $\mathcal{F}$ satisfying

$$
h_{1}(\bar{B}) \cap h_{2}(\bar{B})=\emptyset .
$$

We define the tubes $\overline{\Gamma^{1}}=\Gamma^{\prime}$ and $\overline{\Gamma^{2}}=\left(\Gamma^{\prime}\right)^{* n} * \Gamma^{\prime \prime}$, whose associated holonomy maps are respectively $h_{1}$ and $h_{2}$.

We denote by $H=\left\langle h_{1}, h_{2}\right\rangle$ the semigroup generated by the two holonomy maps of Lemma 13. The action of $H$ on $B$ is a local semigroup version of the action of a Schottky group on $\mathbb{P}^{1}$. In particular there exists a unique closed $H$-invariant set, and it is contained in the closure of every $H$-orbit. This limit set is

$$
\Lambda_{H}:=\bigcap_{n \in \mathbb{N}^{*}}\left(\bigcup_{\left(i_{1}, \ldots, i_{n}\right) \in\{1,2\}^{n}} h_{i_{1}} \circ \cdots \circ h_{i_{n}}(\bar{B})\right) .
$$

The following fairly standard lemma is included for lack of a suitable reference:

Lemma 14. Under the hypothesis of Lemma 13, the set $\Lambda_{H}$ is a Cantor set in B. Moreover, $H$ acts freely discontinuously on $U_{H}=B \backslash \Lambda_{H}$.

Proof. Let $N$ be a natural number and for each finite ordered set $I=\left(i_{n}\right)_{n \leq N} \in\{1,2\}^{N}$ define $h_{I}=h_{i_{1}} \circ \cdots \circ h_{i_{N}} \in H$, and say it has length $N$. By a trivial induction, each $h_{I}: B \rightarrow B$ is an injective holomorphic mapping. We need to analyze the set of points where orbits of the action accumulate, i.e., $\Lambda_{H}=\bigcap_{k} \bigcup_{N(I)=k} h_{I}(B)$. For each $I$ the set $B \backslash h_{I}(B)$ is conformally equivalent to a unique annulus

$$
A_{I}=\left\{z \in \mathbb{C}: r_{I}<|z|<1\right\},
$$


of modulus

$$
m\left(B \backslash h_{I}(B)\right)=-\log \left(r_{I}\right) .
$$

Set $0<c<\min \left\{m\left(B \backslash h_{1}(B)\right), m\left(B \backslash h_{2}(B)\right)\right\}$. We claim that $m\left(B \backslash h_{I}(B)\right)>c N$. Indeed, this can be proved by induction on the length. For $N=1$ it is obvious. Suppose $I^{\prime}$ has length $N-1$ and satisfies $m\left(B \backslash h_{I^{\prime}}(B)\right)>c(N-1)$. Let $I=I^{\prime} i_{N}$. Then by using superadditivity of moduli (see [23, Lemmas 6.3, 6.4, pp. 35-36], or use [1, pp. 10-16], especially Example 3, Theorem 4(b) and Theorem 2) we have

$$
m\left(B \backslash h_{I}(B)\right) \geq m\left(B \backslash h_{I^{\prime}}(B)\right)+m\left(h_{I^{\prime}}(B) \backslash h_{I}(B)\right) .
$$

On the other hand

$$
m\left(h_{I^{\prime}}(B) \backslash h_{I}(B)\right)>m\left(B \backslash h_{i_{N}}(B)\right)>c,
$$

and therefore $m\left(B \backslash h_{I}(B)\right)>c N$.

Hence for an infinite sequence, $I=\left(i_{n}\right)_{n \in \mathbb{N}} \in\{1,2\}^{\mathbb{N}}$, if we consider its truncation $I_{N}$ of length $N$ we find that $\lim _{N \rightarrow \infty} m\left(B \backslash h_{I_{N}}(B)\right)=\infty$ and there exists a unique point $k_{I} \in \bigcap_{n \in \mathbb{N}} h_{i_{1}} \circ \cdots \circ h_{i_{n}}(B) \in B$. It is then clear that the limit set of $H$,

$$
\Lambda_{H}=\bigcup_{I \in\{1,2\}^{\mathbb{N}}} k_{I},
$$

is a totally disconnected, perfect set in $B$, hence a Cantor set.

We now proceed to the proof of Theorem 4. Suppose that we could find an algebraic curve $C$ and a holonomy map $h: B \backslash \Lambda_{H} \rightarrow C$ which is invariant under the action of the semigroup $H$. Then the map $h$ would not extend continuously to any point of $\Lambda_{H}$, and Theorem 4 would follow. While this seems to be asking too much, a slight weakening of this property, by restricting $h$ to an $H$-invariant tree in $B \backslash \Lambda_{H}$, is sufficient for our purposes. The tree structure is already implicit in the multi-indices used to parametrize points of $\Lambda_{H}$ in the proof of Lemma 14. We proceed to constructions of such $h$ based on suspensions using semigroup actions, and containing trees as diagonals. We hope this also provides some understanding of the link to our results in the previous sections.

Consider the bouquet of two oriented circles $Q=S_{1} \vee S_{2}$, with basepoint $*$, and let $\pi_{1}(Q)^{+} \subset \pi_{1}(Q)$ be the positive semigroup generated by the classes $\left[S_{i}\right], i=1,2$, in the fundamental group. Denote by $T$ the Cayley graph of $\pi_{1}(Q)^{+}$associated to the set of generators $\left[S_{1}\right],\left[S_{2}\right]$; this is a dyadic rooted tree and $\pi_{1}(Q)^{+}$acts on $T$ by the restriction of the $\pi_{1}(Q)$ action on its Cayley graph. We will denote by $\rho: \pi_{1}(Q)^{+} \rightarrow H$ the representation defined by sending $\left[S_{i}\right]$ to $h_{i}$, which clearly gives a semigroup isomorphism. The next proposition provides in particular the tree in $B \backslash \Lambda_{H}$, on which our suspension analogy will be built.

Proposition 15. There exist two continuous maps $F_{i}: T \rightarrow \mathbb{P}^{2}, i=1,2$, such that

(1) $F_{0}$ takes values in $U_{H}=B \backslash \Lambda_{H}$ and is $\rho$-equivariant,

(2) $F_{1}$ takes values in a smooth algebraic curve $C$ of $\mathbb{P}^{2}$ and is $\pi_{1}(Q)^{+}$-invariant, thus giving an immersed image of $Q$,

(3) $F_{0}$ and $F_{1}$ are $\mathcal{F}$-homotopic. 
Assuming this proposition, let us prove Theorem 4. Denote by $h: U \subset B \rightarrow V \subset C$ a germ of holonomy map defined by the $\mathcal{F}$-homotopy between $F_{0}$ and $F_{1}$. Note that by equivariance each point $q \in \Lambda_{H}$ can be approached by an infinite path in $F_{0}(T)$ that, apart from continuity, has very little regularity at $q$ (it is essentially log-fractal, i.e. fractal with respect to the $\log (r)$ transformation of polar to cylindrical coordinates). Nevertheless, along such a path, the holonomy $h$ will not have any limit, since it is a curve winding infinitely many times around the two circles of the bouquet $F_{1}(T)$. (Recall the discussion of asymptotic values following Proposition 9.) Thus $\Lambda_{H}$ is a subset of the set of singularities for the topological continuation of $h$, reducing the proof of Theorem 4 to Proposition 15, which we will now consider. For the proof of the latter we will first produce a pair $F_{0}, F_{1}$ with all the desired properties except that $F_{1}$ does not take values in an algebraic curve. A basic approximation method will allow us to correct this easily.

To guarantee the continuity of some of the constructions we will repeatedly use the fact that, by work of Lins-Neto [25, Theorem 1, p. 353], for the class of foliations in Theorem 4 , the leaves of $\mathcal{F}$ are of hyperbolic type and the unique complete metric of constant negative curvature in each leaf is continuous in $\mathbb{P}^{2} \backslash \operatorname{Sing}(\mathcal{F})$ (the two conclusions hold as soon as the foliation has degree greater than 2 and nondegenerate singularities). This allows one, given a leafwise path, to homotope it, fixing endpoints, to the unique geodesic representative in its class.

Let $p$ be a point of $U_{H}=B \backslash \Lambda_{H}$. The basic technical device is a pair $\gamma=\left(\gamma^{1}, \gamma^{2}\right)$ of continuous families $\gamma^{i}=\left\{\gamma_{t}^{i}(s)\right\}_{t \in[0,1]}$ of $s$-leafwise paths satisfying

- $\gamma_{0}^{i}$ is the constant path at $p$,

- $\gamma_{t}^{i}(0) \in U_{H}$ and $\gamma_{1}^{i}(0)=h_{i}(p)$,

- $\gamma_{1}^{i}$ is the leafwise geodesic path starting at $h_{i}(p)$ and ending at $p$, and carrying the holonomy map $h_{i}^{-1}$.

To ensure existence of such families, for $i=1,2$, we add the special condition:

$$
\left\{\gamma_{t}^{i}(1): t \in[0,1]\right\} \subset\left\{\gamma_{t}^{i}(0): t \in[0,1]\right\} \cup\left\{\gamma_{1}^{i}(s): s \in[0,1]\right\},
$$

i.e. start with the constant path at $p$, and begin moving continuously to the constant path at $h_{i}(p)$ in the space of $U_{H}$-valued constant paths. Then, we construct a homotopy (with one free extremity) between the constant path at $h_{i}(p)$ and the leafwise geodesic path going from $h_{i}(p)$ to $p$.

Given $\gamma$, we now indicate how to construct associated continuous maps $F_{j}^{\gamma}: T \rightarrow \mathbb{P}^{2}$, for $j=0,1$. For $i=1,2$, denote by $\tau_{i}:[0,1] \rightarrow U_{H}$ the path $\tau_{i}(t)=\gamma_{t}^{i}(0)$ going from $p$ to $h_{i}(p)$. We extend the $\tau_{i}$ 's as a continuous $\rho$-equivariant map $F_{0}^{\gamma}: T \rightarrow B$. We refer to its image as the tree generated by $\tau_{1} \vee \tau_{2}$. Notice that for each point $q \in F_{0}^{\gamma}(T)$ there exist unique $i \in\{1,2\}, t \in[0,1)$ and a finite composition $h_{q}$ of $h_{1}$ and $h_{2}$ 's of maximal length such that

$$
h_{q}\left(\tau_{i}(t)\right)=q .
$$

Parametrize $Q$ by $[0,1] \sqcup[0,1]$ with all endpoints identified. Now, define for $i=1,2$, $\sigma_{i}:[0,1] \rightarrow \mathbb{P}^{2}$ as the map $\sigma_{i}(t)=\gamma_{t}^{i}(t)$. Obviously $\sigma_{i}(0)=\sigma_{i}(1)=p$. Thus, we can 


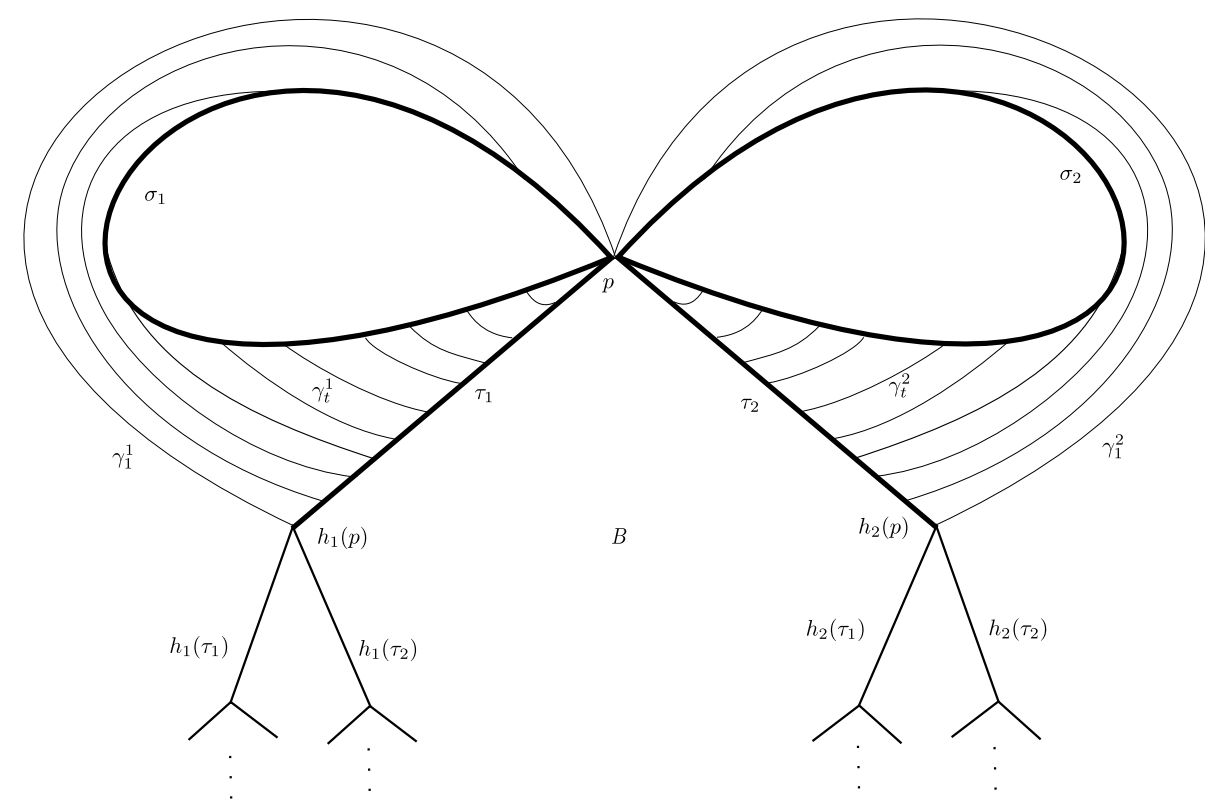

Fig. 2. The $\mathcal{F}$-homotopy between $\tau_{i}$ and $\sigma_{i}$ is realized by the finer paths.

define a map $\sigma_{1} \vee \sigma_{2}$ from $Q$ to $\mathbb{P}^{2}$, which will be referred to as the diagonal. Then we obtain a $\pi_{1}(Q)^{+}$-invariant continuous map $F_{1}^{\gamma}: T \rightarrow \mathbb{P}^{2}$ by composing $\sigma_{1} \vee \sigma_{2}$ with the restriction to $T$ of the universal covering map of $Q$.

The family $\gamma$ provides an $\mathcal{F}$-homotopy between generators $\tau_{1} \vee \tau_{2}$ of the tree and the generators $\sigma_{1} \vee \sigma_{2}$ of the diagonal (see Figure 2). We will now extend this homotopy and get an $\mathcal{F}$-homotopy between $F_{0}^{\gamma}$ and $F_{1}^{\gamma}$. The basic idea is that, by using (4.1), any point $q$ in the tree can be joined by a leafwise path in the tube of $h_{q}^{-1}$ (a concatenation of the geodesic tubes of $h_{1}^{-1}$ and $h_{2}^{-1}$ ) to its corresponding point $\tau_{i}(t)$. Note that we follow the tubes in the expanding direction of the holonomy. By this procedure we obtain a family of leafwise paths parametrized by $T$ which is continuous only outside the vertices of $T$. At the vertices of the tree the number of tubes of $h_{1}^{-1}$ or $h_{2}^{-1}$ that we follow changes by 1 because of the conditions defining $h_{q}$. However, if for each $q$ in the tree instead of considering the leafwise path following the expanding tubes until $\tau_{i}(t)$ we go a little further by concatenating the path $\gamma_{t}^{i}$, the family will also be continuous at the vertices, since $\gamma_{1}^{i}$ and the leafwise path in the tube of $h_{i}^{-1}$ are both the same geodesic. The endpoints of the new family will be in the diagonal instead of being in $B$.

To avoid cumbersome notations of homotopy parametrizations we have preferred to represent the homotopy between $F_{0}^{\gamma}$ and $F_{1}^{\gamma}$ by a figure. For each $i=1,2$ consider the holonomy tube $\Gamma^{i}:[0,1] \times h_{i}(B) \rightarrow \mathbb{P}^{2}$ whose associated holonomy map is $h_{i}^{-1}$, given by Lemma 13. To get continuity it is important here to use the leafwise geodesics for the $\Gamma^{i}(\cdot, q)$ 's. We find those tubes and the family $\gamma$ in Figure 3 concatenated in the 


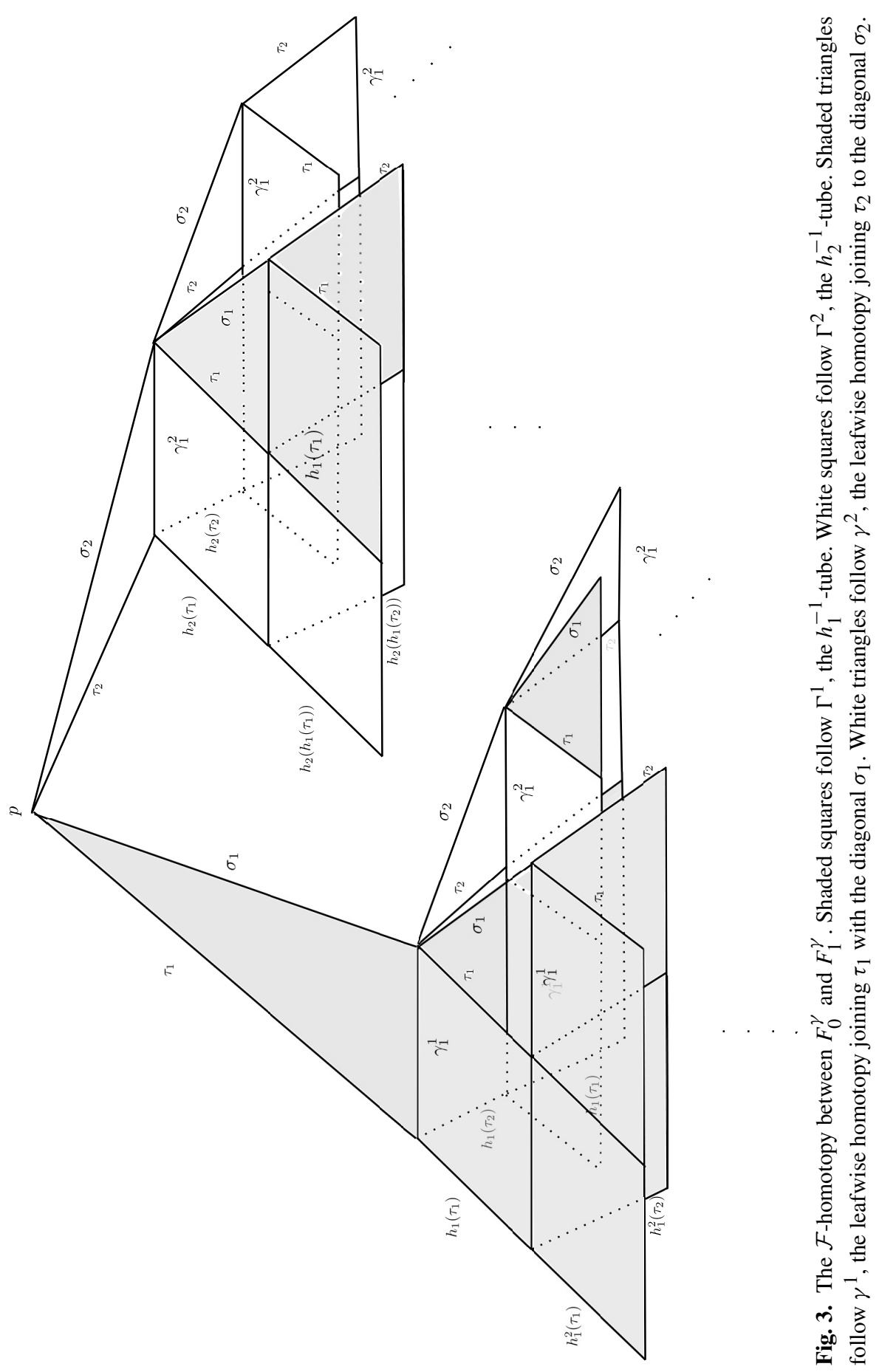


appropriate way to produce the desired $\mathcal{F}$-homotopy between $F_{0}^{\gamma}$ and $F_{1}^{\gamma}$. Formally, the existence proof uses a straightforward induction on the heights of edges (i.e. lengths of multiindices) in the tree.

Some remarks are in order at this point:

First, the choice of the pair $\gamma=\left(\gamma^{1}, \gamma^{2}\right)$ could have been done by using other more differential-topological conditions instead of using the metric arguments that apply only under the hypothesis that all leaves are of hyperbolic type. These other choices allow us to guarantee the continuity of the $\mathcal{F}$-homotopies to more general foliations.

Second, let us relate the diagonal construction of Section 3.2 in the Riccati context to the present context. In the former case the diagonal can be interpreted in the covering space, which is a product, as the graph of a holomorphic map from a leaf to a transversal. The asymptotical values of this map provide singular points for the holonomy germ determined by a germ of its inverse. In other words there are infinite paths in the diagonal whose projections to the fibre converge, while the projection onto the leaf goes to infinity. In the present case the diagonal is represented by the map $F_{1}^{\gamma}: T \rightarrow \mathbb{P}^{2}$. The basic point is that the diagonal can be projected to the tree $F_{0}^{\gamma}(T)$ in the transversal $B$ and also to a tree in the leaf of $\mathcal{F}$ through $p$, by using the holonomy tubes of $h_{1}$ and $h_{2}$ to define lifts. If we take an infinite path in $T$ to a point in the boundary, its projection from the diagonal to $B$ converges, while its projection onto the leaf diverges.

The proof is complete except that $F_{1}^{\gamma}$ constructed above does not necessarily take values in an algebraic curve of $\mathbb{P}^{2}$, so we now modify the tubes $\gamma$ to obtain this latter condition. For this purpose, we will construct in what follows a perturbation $\hat{\sigma}_{1} \vee \hat{\sigma}_{2}$ of $\sigma_{1} \vee \sigma_{2}$ in the uniform topology, based at the point $p$, so that its image is contained in an algebraic curve. Then the construction of a pair $\hat{\gamma}=\left(\hat{\gamma}_{1}, \hat{\gamma}_{2}\right)$ whose corresponding diagonal is $\hat{\sigma}_{1} \vee \hat{\sigma}_{2}$ follows by a simple perturbation of the construction above. One should note that the perturbation $\hat{\sigma}_{1} \vee \hat{\sigma}_{2}$ determines uniquely a perturbation of $\tau_{i}$ compatible with the $\gamma^{i}$ construction, which then makes the continuous dependence of the $\gamma^{i}$ quite obvious.

Lemma 16. There exists a continuous mapping $\hat{\sigma}_{1} \vee \hat{\sigma}_{2}: Q \rightarrow \mathbb{P}^{2}$ arbitrarily close to $\sigma_{1} \vee \sigma_{2}$ in the uniform topology, whose image is contained in a smooth algebraic curve of $\mathbb{P}^{2}$, and both maps share the same basepoint $p \in \mathbb{P}^{2}$.

Proof. Let $\varepsilon>0$. For $i=1,2$ choose a $C^{\infty}$ map $\tilde{\sigma}_{i}: S^{1} \rightarrow \mathbb{C}^{2} \subset \mathbb{P}^{2}$ in the $\varepsilon / 4$ neighbourhood of $\sigma_{i}$ satisfying $\sigma_{i}(1)=\tilde{\sigma}_{i}(1)=p$. We can assume that $\tilde{\sigma}_{1}^{\prime}(1), \tilde{\sigma}_{2}^{\prime}(1)$ are $\mathbb{C}$-linearly independent. The Fourier series of $\tilde{\sigma}_{i}$ (resp. the series' first derivative) converges uniformly to $\tilde{\sigma}_{i}$ (resp. $\tilde{\sigma}_{i}^{\prime}$ ) so by truncating the series for some sufficiently large $N>0$ and applying a small translation we get a finite family $\left\{a_{n}^{i}, b_{n}^{i} \in \mathbb{C}:|n|<N\right\} \subset \mathbb{C}$ and a map

$$
\bar{\sigma}_{i}\left(e^{2 i \pi t}\right)=\left(\sum_{|n|<N} a_{n}^{i}\left(e^{2 i \pi t}\right)^{n}, \sum_{|n|<N} b_{n}^{i}\left(e^{2 i \pi t}\right)^{n}\right),
$$

which is in the $\varepsilon / 2$-neighbourhood of $\sigma_{i}$ and satisfies $\bar{\sigma}_{i}(1)=p$. The map $\bar{\sigma}_{i}$ is the restriction of a rational map $\mathbb{P}^{1} \rightarrow \mathbb{P}^{2}$ to $S^{1} \subset \mathbb{P}^{1}$ so its image is contained in an algebraic curve $C_{i}=\left\{f_{i}=0\right\} \subset \mathbb{P}^{2}$ that contains $p$. The noncollinearity of $\tilde{\sigma}_{1}^{\prime}(1)$ and $\tilde{\sigma}_{2}^{\prime}(1)$ guarantees that $p$ is a normal crossing point of the reducible curve $C_{1} \cup C_{2}=\left\{f_{1} f_{2}=0\right\}$. 
For a generic and sufficiently small $\delta \in \mathbb{C}$, the curve $\tilde{C}=\left\{f_{1} f_{2}=\delta\right\}$ is smooth and $\varepsilon / 2$ close to $C_{1} \cup C_{2}$. In a neighbourhood of $p, \tilde{C}$ is arc connected (it is biholomorphic to a disc with a finite number of holes) so we can find a point $q \in \tilde{C}$ which is $\varepsilon / 2$-close to $p$ and two loops $\breve{\sigma}_{1}, \breve{\sigma}_{2}$ in $\tilde{C}$ with basepoint at $q$ which are $\varepsilon / 2$-close to $\sigma_{1}$ and $\sigma_{2}$ respectively. By choosing an automorphism $\varphi$ of $\mathbb{P}^{2}$ which is $\varepsilon / 4$-close to the identity sending $q$ to $p$ we conclude that the curve $C:=\varphi(\tilde{C})$ and the loops $\hat{\sigma}_{i}:=\varphi \circ \breve{\sigma}_{i}$ have the desired properties.

Recall that there is an open and dense set in the quasi-projective manifold of degree $d$ foliations of $\mathbb{P}^{2}$ such that no foliation belonging to that set has an algebraic curve (see [24, Theorem B, p. 196]). It is rather classical to show that there is also a dense open set such that every foliation belonging to this set has only singularities with $\lambda_{2} / \lambda_{1}$ nonreal, where $\lambda_{1}, \lambda_{2}$ are the eigenvalues (see [26]). Hence, a generic foliation satisfies the hypothesis of Theorem 4.

\subsection{Proof of Theorem 5}

A careful analysis of the proof of Theorem 4 shows that if $B$ is a transverse disc to a holomorphic singular foliation $\mathcal{F}$ of $\mathbb{P}^{2}$ having only hyperbolic leaves and $h_{1}, \ldots, h_{l}$ : $B \rightarrow B$ are holonomy maps such that

- there exists a constant $c<1$ satisfying $\left|h_{i}^{\prime}(x)\right|<c$ for all $x \in B$ and $i \in\{1, \ldots, l\}$, and

- each $h_{i}$ carries a holonomy tube,

then we can find an algebraic curve $C$ and a germ of holonomy of $\mathcal{F}$ from $B$ to $C$ for which every point in the limit set of the action of the semigroup $H$ generated by $h_{1}, \ldots, h_{l}$ on $B$, namely

$$
\Lambda_{H}=\bigcup_{\left(i_{n}\right) \in\{1, \ldots, l\}^{\mathbb{N}}}\left(\bigcap_{n \geq 0} h_{i_{1}} \circ \cdots \circ h_{i_{n}}(B)\right),
$$

is a singularity for its analytic continuation. In fact we only need to remark that the leafwise Poincaré metric is continuous [25], adapt Proposition 15 by taking $T$ as an $l$-adic tree instead of a dyadic tree, $Q$ as a bouquet of $l$ circles, and use an adaptation of Lemma 16 to guarantee that as close to a continuous map $Q \rightarrow \mathbb{P}^{2}$ as we wish, there is another such map with image contained in an algebraic curve.

The plane algebraic foliations that will satisfy Theorem 5 are examples that we learned from Loray and Rebelo's paper [28]. To find the admissible germ for such a foliation, we will construct a semigroup in the holonomy pseudogroup, defined by some holomorphic maps carried by holonomy tubes, whose limit set has nonempty interior, as above. For this purpose, we review Loray/Rebelo's techniques.

The important property satisfied by these foliations is that their pseudogroup is nondiscrete: namely, there exist transverse discs $B \subset B^{\prime}$, with $B$ relatively compact in $B^{\prime}$, and a sequence of holonomy maps $g_{n}: B \rightarrow B^{\prime}$ which are different from the identity for every integer $n$, but nevertheless converge to the identity uniformly in $B$. The main ingredient to ensure that a foliation has a nondiscrete pseudogroup is [28, Lemma 3.3]: 
Lemma 17. There is a constant $\varepsilon_{0}>0$ such that the following holds. Let $f: \mathbb{D} \rightarrow \mathbb{D}$ be a map defined by $f(z)=\lambda z$ for some complex number $\lambda$ of modulus $|\lambda|<1$ and with $|\lambda-1| \leq \varepsilon_{0}$. Let $g: \mathbb{D} \rightarrow \mathbb{C}$ be an injective holomorphic map, $\varepsilon_{0}$-close to the identity, i.e. $\sup _{z \in \mathbb{D}}|g(z)-z| \leq \varepsilon_{0}$. Then there exists an integer $N \geq 1$ such that the holomorphic maps defined by $g_{0}=g$ and $g_{k+1}=f^{-N} \circ\left[f, g_{k}\right] \circ f^{N}$ for every integer $k \in \mathbb{N}$ have domain of definition at least the disc $\mathbb{D}_{1 / 3}$, and converge uniformly to the identity as $k \rightarrow \infty$. Moreover, if $g$ is not an affine map, then $g_{k}$ is different from the identity for every $k$.

In what follows, we introduce the set $\mathcal{U}_{d}$ of degree $d$ plane algebraic foliations, whose pseudogroup contains two maps $f$ and $g$ defined on some transversal $B \simeq \mathbb{D} \subset B^{\prime} \simeq \mathbb{C}$ as in Lemma 17, and such that moreover:

- $\lambda$ is not real,

- $g(0)$ is different from 0 ,

- $g$ is not affine in the linearization coordinate of $f$,

- there exist holonomy tubes carrying the maps $f$ and $g$,

- the singularities are of hyperbolic type, and the foliation does not carry any invariant algebraic curve. In particular, the leaves are hyperbolic, and the Poincaré metric on the leaves is continuous.

All the above conditions are open (there is a little work to see that after perturbation $g$ is still not affine in the linearization coordinate of $f$; this is due to the fact that this coordinate depends continuously on $f$ ); hence $\mathcal{U}_{d}$ is an open set. Loray/Rebelo [28] proved that this set is not empty. The idea is to construct a particular foliation satisfying all the conditions but the last one, and to perturb it so that the singularities become hyperbolic, and no algebraic curve is invariant. Such foliations are for instance the Riccati foliations with dense monodromy group: the reader can check that they satisfy all the conditions except the last one.

At this point, an important thing to observe is that if a foliation belongs to $\mathcal{U}_{d}$, the corresponding maps $g_{k}$ defined on $\mathbb{D}_{1 / 3}$ are carried by holonomy tubes for every $k$. These tubes are obtained by concatenating the tubes of the maps $f, g, f^{-1}, g^{-1}$.

Next, we review another fundamental result proved in Loray/Rebelo's paper: the existence of flows in the closure of the pseudogroup of a foliation in $\mathcal{U}_{d}$. Here is the precise statement (see [28, Corollary 4.2]):

Lemma 18. Let $\mathcal{F}$ be a foliation belonging to $\mathcal{U}_{d}$ and $\mathcal{G}$ be the holonomy pseudogroup of $\mathcal{F}$. Then there is a transversal $B$ isomorphic to the unit disc, and two holomorphic vector fields $X_{1}, X_{2}$ defined on $\mathbb{D}$, such that $X_{1}(0)$ and $X_{2}(0)$ are independent over the real numbers, and such that for every small enough real number $t$, the map $\exp \left(t X_{i}\right)$ is defined on the disc $\mathbb{D}_{1 / 2}$ (with values in $\mathbb{D}$ ) and is the uniform limit of a sequence $k_{n}$ of elements of $\mathcal{G}$ on $\mathbb{D}_{1 / 2}$ (in particular the domain of definition of $k_{n}$ contains $\mathbb{D}_{1 / 2}$ for every $n)$. Moreover, the maps $k_{n}$ are associated to holonomy tubes.

Note that Loray/Rebelo's Corollary 4.2 states that there is at least one vector field $X_{1}$ satisfying the conclusion of Lemma 18 such that $X_{1}(0) \neq 0$. Then, by taking $X_{2}=f_{*} X_{1}$, 
we get another vector field, and these two are as desired. Notice also that the maps $k_{n}$ are carried by holonomy tubes. Indeed, they are constructed by considering compositions of the form $f^{-N_{k}} \circ g_{k} \circ f^{N_{k}}$, for some integer $N_{k}$, where $g_{k}$ are the maps constructed in Lemma 17. These maps are as above carried by holonomy tubes: the concatenations of the holonomy tubes of the maps $f, f^{-1}$ and $g_{k}$.

The following lemma (together with the construction sketched above) finishes the proof:

Lemma 19. Let $\mathcal{F}$ be a foliation as in Lemma 18. Then there exist a finite number of holonomy maps $h_{1}, \ldots, h_{l}$ from a transverse disc $B \simeq \mathbb{D}$ to itself, carried by holonomy tubes, such that the limit set of the action of the semigroup $H$ generated by the $h_{i}$ 's on $\mathbb{D}$ has nonempty interior. Moreover the $h_{i}$ 's can be chosen to be contracting, i.e. there is a constant $0<c<1$ such that $\left|h_{i}^{\prime}(x)\right|<c$ for all $i=1, \ldots$, l and $x \in \mathbb{D}$.

Proof. Let $h_{1}, \ldots, h_{l}: \mathbb{D} \rightarrow \mathbb{D}$ be some maps and $H$ the semigroup generated by them. Suppose that there is a point $p$ in $\mathbb{D}$, and an infinite sequence of indices $\left\{i_{1}, i_{2}, \ldots\right\} \subset$ $\{1, \ldots, l\}$ such that we can define the sequence of points $p_{1}=h_{i_{1}}^{-1}(p)$ and $p_{n}=$ $h_{i_{n}}^{-1}\left(p_{n-1}\right)$ for every $n>1$. If we suppose further that for every $i=1, \ldots, l$ and $x \in \mathbb{D}$, $\left|h_{i}^{\prime}(x)\right|<c<1$ then actually $p=\bigcap_{n \geq 1} h_{i_{1}} \circ \cdots \circ h_{i_{n}}(\mathbb{D})$. By definition $p$ is a limit point of the semigroup. Our goal will be to find such maps $h_{1}, \ldots, h_{l}$ in the holonomy pseudogroup $\mathcal{G}$ of $\mathcal{F}$, defined on $\mathbb{D}$, and such that for every point in a nonempty open set $U$ of $\mathbb{D}$ we can find a sequence $i_{1}, i_{2}, \ldots$ as above.

Observe that because $X_{1}$ and $X_{2}$ are not collinear at 0 , there is a neighbourhood of 0 , say $\mathbb{D}_{r}$ with $r>0$, and a $\tau>0$, such that for every point $p$ of $\overline{\mathbb{D}_{r}}$, there are unique real numbers $t_{1}, t_{2}$ such that $\left|t_{1}\right|,\left|t_{2}\right|<\tau$ and $0=\exp \left(t_{1} X_{1}\right) \exp \left(t_{2} X_{2}\right)(p)$. Moreover, we can suppose that the composition $\exp \left(t_{1} X_{1}\right) \exp \left(t_{2} X_{2}\right)$, and its inverse $\exp \left(-t_{2} X_{2}\right) \exp \left(-t_{1} X_{1}\right)$, are defined on $\mathbb{D}_{1 / 4}$ with values in $\mathbb{D}$, for every $\left|t_{1}\right|,\left|t_{2}\right|<\tau$.

We fix some constants. Denote $M>0$ an upper bound for all the derivatives

$$
\left|\left(\exp \left(-t_{2} X_{2}\right) \exp \left(-t_{1} X_{1}\right)\right)^{\prime}(p)\right|
$$

for every point $p$ in the ball of radius $1 / 4$ and every $\left|t_{1}\right|,\left|t_{2}\right| \leq \tau$. Let $k$ be an integer such that $|\lambda|^{k}<\min (r, 1 / 8,1 / 2 M)$.

Given $p \in \overline{\mathbb{D}_{r}}$, we approximate the maps $\exp \left(t_{j} X_{j}\right), j=1,2$, on $\mathbb{D}_{1 / 2}$ by elements $k_{j}$ of the pseudogroup defined on $\mathbb{D}_{1 / 2}$, so that the map $g=k_{1} k_{2}$ is defined on $\mathbb{D}_{1 / 8}$ with derivative bounded by $2 M$, and $g(p)$ belongs to the disc $\mathbb{D}_{|\lambda|^{k}}$. If $k_{j}$ is sufficiently close to $\exp \left(t_{j} X_{j}\right)$, we will also get that $g^{-1}$ is defined on $\mathbb{D}_{1 / 8}$, and that its derivative is bounded by $2 M$.

By compactness of $\overline{\mathbb{D}_{r}}$, we can find a finite number of elements $g_{1}, \ldots, g_{l}$ of $\mathcal{G}$ defined on $\mathbb{D}_{1 / 8}$ such that for every point $p$ in $\mathbb{D}_{r}$, there is an index $i \in\{1, \ldots, l\}$ such that $g_{i}(p)$ belongs to $\mathbb{D}_{|\lambda|^{k} r}$. Moreover, the maps $g_{i}$, together with their inverses, are defined on $\mathbb{D}_{1 / 8}$ with derivatives bounded by $2 M$.

Consider the maps $h_{i}=g_{i}^{-1} \circ f^{k}$ for $i=1, \ldots, l$. Because $|\lambda|^{k}<1 / 8, h_{i}$ is a map from $\mathbb{D}$ to itself. Moreover, the derivative of $h_{i}$ is uniformly bounded by $2 M|\lambda|^{k}<1$. We have $h_{i}^{-1}=f^{-k} \circ g_{i}$, i.e. $h_{i}^{-1}=\lambda^{-k} g_{i}^{-1}$. Since for every $p \in U=\mathbb{D}_{r}$ there is an 
index $i \in\{1, \ldots, l\}$ such that $g_{i}(p)$ belongs to $\mathbb{D}_{|\lambda|^{k} r}$ we infer that $h_{i}^{-1}(p)$ belongs to $U$. We define $i_{1}:=i$ and repeat the argument for $h_{i_{1}}^{-1}(p)$. By induction we get our sequence $i_{1}, i_{2}, \ldots$ The proof is complete.

\section{Afterword}

We proved that, in the presence of rich contracting dynamics for a holomorphic foliation $\mathcal{F}$ of $\mathbb{P}^{2}$ (which is known to be a generic property), there are special choices of an algebraic curve $T$ generically transverse to $\mathcal{F}$ such that some holonomy correspondence has a maximal domain of definition with large boundary. We produced examples whose singular sets contain Cantor sets of positive dimension, and even open sets. This disproves Loray's Conjecture, but does not exclude refining the problem to that of finding a better choice for $T$.

Our choices of $T$, which would seem pathological in view of the conjectures cited in the introduction, reflect the fact that foliations with sufficienly rich holonomy have special transversals, which we called diagonals, indicating perhaps a hidden duality involving the holonomy action on a transversal, and the pseudogroup of transition functions along the leaves. More precisely, we found some part of the foliation which can be described as a suspension, where the monodromy has a nontrivial domain of discontinuity when acting on the transverse space, with quotient identifying with the base (and the diagonal). So it is not yet clear whether the choices of diagonal $T$ should be regarded as counterexamples in a project aiming at finding fibre-like transversals, or rather viewed as an important aspect of the structure of $\mathcal{F}$.

Basic examples of these non-fibre-like transverse lines arise notably in the setting of Riccati equations, where, as explained before, we have a suspension foliation with linear fibres, and thus no lack of good transverse lines, realizing holonomy as a group of automorphisms.

The suspension construction behind the first part of Theorem 2 illustrates the nontrivial nature of the choice of $T$ (even when constrained to be linear as in [27]) and suggests the need for further research.

Recall that this suspension construction, for a circle bundle over a Riemann surface, is also the most basic source of examples of Levi-flat hypersurfaces. This is no coincidence; the singular sets for holonomy extension in this article are associated to laminations within the holomorphic foliation (albeit laminations with real 1-dimensional leaves, modelled on the product of a Cantor set by a tree, in the generic context of Theorem 4). We recall that such suspensions and diagonals have also been considered from the viewpoint of Stein spaces and harmonic sections by [9]. The birational maps that we realize of such suspensions into $\mathbb{P}^{2}$ thus provide singular Levi-flat hypersurfaces with rich dynamics in $\mathbb{P}^{2}$, as well as interesting examples of Stein spaces, and may provide further insight into the general properties of Levi-flat hypersurfaces in $\mathbb{P}^{2}$.

Note that the natural boundary here is associated to the Levi-flat boundary of a domain in $\mathbb{P}^{2}$. The constructions also provide examples of topologically trivial, but analytically nontrivial deformations of foliations of $\mathbb{P}^{2}$ also admitting Levi-flat hypersurfaces. They 
constitute counterexamples to a conjecture by Scárdua asserting that every holomorphic foliation of $\mathbb{P}^{2}$ having an invariant line is either Darboux integrable or absolutely rigid (see [19] for details). Indeed, for each $p \in \mathbb{C} \backslash\{0,1\}$ we can consider the Riccati foliation $\mathcal{F}_{p}$ on the Hirzebruch surface $\mathbb{F}_{1}$ associated to the uniformizing structure of the 4-punctured sphere $\Sigma_{p}=\mathbb{C} \backslash\{0,1, p\}$ as in Section 3. The analytical type of $\mathcal{F}_{p}$ changes as the cross ratio of the position of the invariant fibres, i.e. $p$, changes. However for sufficiently close values of $p$ two such foliations are equivalent by a fibred homeomorphism that is close to the identity.

We point out that we do not know whether or not there exists a holomorphic foliation of the projective plane and an admissible germ from an algebraic curve to itself which has a natural boundary. Also, we do not know how many singularities the analytic continuation of the holonomy of two generic lines in a generic foliation may have (taking, for example, the holonomy along the trivial path at the intersection of the two lines).

Another interesting problem is to determine the difference between the Riemann surface of a holonomy correspondence obtained by analytic continuation, and the maximal domain where the map is a holonomy map. We found that in foliations defined by closed 1-forms, for any holonomy germ between rational curves, these two domains coincide, up to a countable number of points. However, it is conceivable that there exists a holonomy correspondence whose domain of holonomy is considerably smaller: consider $k$-fold coverings ramified along the diagonal, $\Delta$ of $S_{+}=\left(\widetilde{\Sigma} \times \mathbb{H}^{2}\right) / \pi_{1}(\Sigma) \subset S$ (taking $k=$ the Euler class of the circle bundle $=c_{1}(N \Delta)$, up to sign, ensures existence) or of any such suspension type disc bundles with holomorphic diagonal, and extend a bit in $S$, beyond the Levi-flat boundary $\partial S_{+}$. Then the leaf space of the cover over $S_{+}$is just the disc; i.e. the diagonal (in the cover), or the fibre $\mathbb{H}^{2}$ in $S_{+}$, but on the part of the cover beyond $S_{+}$ the leaf space of the cover is the $k$-fold cover of the annulus outside of $S^{1}=\partial \mathbb{H}^{2} \subset \mathbb{P}^{1}$. Unfortunately we are unable at this time to provide such examples in the algebraic context. This non-Hausdorff leaf space structure is closely related to considerations in the real codimension one case as studied by Palmeira [31], but it is not at all clear what analogous structures one might find for singular holomorphic foliations of the complex projective plane.

While one might be tempted by a certain optimism as regards the bridges connecting analytic holonomy extension to techniques from the topology of real codimension one foliations, or the PDE theory of holomorphic extensions, we should add that the subject seems to be fraught with as many hidden traps as temptations, and should be approached with a certain degree of caution.

Acknowledgments. We thank Yuli Ilyashenko, David Marín and Paulo Sad, as well as Andrzej Żuk, for interesting discussions during the preparation of this work and the referee for a careful reading. The authors would also like to thank the institutions CRM (Bellaterra), UNAM (DF y Cuernavaca), IMPA (Rio de Janeiro) for their hospitality. G. Calsamiglia acknowledges support from FAPERJ/CNPq, B. Deroin from the Ministerio de Educación y Ciencia de España, ANR08-JCJC-0130-01 (France), ANR-09-BLAN-0116 (France) and from the International Cooperation Agreement Brazil-France, A. Guillot from CONACyT (Mexico) grant 58354 and PAPIIT-UNAM (Mexico) grant IN102307. S. Frankel is also grateful to the Max Planck Institute for Mathematics in the Sciences, where part of this work was done. 


\section{References}

[1] Ahlfors, L. V.: Lectures on Quasiconformal Mappings. Van Nostrand-Reinhold, Princeton (1966). Zbl 0138.06002 MR 0200442

[2] Barth, W., Hulek, K., Peters, C., Van de Ven, A.: Compact Complex Surfaces. 2nd ed., Springer, Berlin (2004) Zbl 1036.14016 MR 2030225

[3] Belliart, M., Liousse, I., Loray, F.: The generic rational differential equation $d w / d z=$ $P_{n}(z, w) / Q_{n}(z, w)$ on $\mathbb{P}^{2}$ carries no interesting transverse structure. Ergodic Theory Dynam. Systems 21, 1599-1607 (2001) Zbl 1018.37025 MR 1869061

[4] Bonatti, C., Langevin, R., Moussu, R.: Feuilletages de $\mathbf{C P}(n)$ : de l'holonomie hyperbolique pour les minimaux exceptionnels. Publ. Math. Inst. Hautes Études Sci. 75, 123-134 (1992) Zbl 0782.32023 MR 1179078

[5] Brunella, M.: Birational Theory of Foliations. Monografías de Matemática, Instituto de Matemática Pura e Aplicada, Rio de Janeiro (2000) Z Zbl 1073.14022 MR 1948251

[6] Camacho, C., Lins Neto, A., Sad, P.: Minimal sets of foliations on complex projective spaces. Publ. Math. Inst. Hautes Études Sci. 68, 187-203 (1988) Z Zbl 0682.57012 MR 1001454

[7] Cruz López, M.: Dynamics of piecewise conformal automorphisms of the Riemann sphere. Ergodic Theory Dynam. Systems 25, 1767-1774 (2005) Zbl 1085.37041 MR 2183292

[8] Culler, M., Shalen, P. B.: Varieties of group representations and splitting of 3-manifolds. Ann. of Math. 117, 109-146 (1983) Zbl 0529.57005 MR 0683804

[9] Diederich, K., Ohsawa, T.: Harmonic mappings and disc bundles over compact Kähler manifolds. Publ. RIMS Kyoto Univ. 21, 819-833 (1985) Zbl 0601.32023 MR 0817167

[10] Françoise, J.-P., Roytvarf, N., Yomdin, Y.: Analytic continuation and fixed points of the Poincaré mapping for a polynomial Abel equation. J. Eur. Math. Soc. 10, 543-570 (2008) Zbl 1140.30015 MR 2774340

[11] Gallo, D., Kapovich, M., Marden, A.: The monodromy groups of Schwarzian equations on closed Riemann surfaces. Ann. of Math. (2) 151, 625-704 (2000) Zbl 0977.30028 MR 1765706

[12] Goldman, W.: Geometric structures on manifolds and varieties of representations. In: Geometry of Group Representations (Boulder, CO, 1987), Contemp. Math. 74, Amer. Math. Soc., 169-198 (1988) Zbl 0659.57004 MR 0957518

[13] Guillot, A.: Sur les équations d'Halphen et les actions de $\mathrm{SL}_{2}(\mathbb{C})$. Publ. Math. Inst. Hautes Études Sci. 105, 221-294 (2007) Zbl 1141.32007 MR 2354208

[14] Hille, E.: Ordinary Differential Equations in the Complex Domain. Pure Appl. Math., WileyInterscience, New York (1976) Zbl 0343.34007 MR 0499382

[15] Hussenot, N.: Mouvement Brownien appliqué à l'étude de la dynamique des feuilletages transversalement holomorphes. Thèse, Univ. de Nantes (2012)

[16] Ilyashenko, Yu.: Centennial history of Hilbert's 16th problem. Bull. Amer. Math. Soc. (N.S.) 39, 301-354 (2002) Zbl 1004.34017 MR 1898209

[17] Ilyashenko, Yu.: Some open problems in real and complex dynamical systems. Nonlinearity 21, 101-107 (2008) Zbl 1183.37016 MR 2425322

[18] Ilyashenko, Yu.: Persistence theorems and simultaneous uniformization. Proc. Steklov Inst. Math. 254, 184-200 (2006) MR 2301006

[19] Ilyashenko, Yu., Moldavskis, V.: Total rigidity of generic quadratic vector fields. Moscow Math. J. 11, 521-530 (2011) Zbl pre06126147 MR 2894428

[20] Ilyashenko, Yu., Shcherbakov, A. A.: On skew cylinders and simultaneous uniformization. Proc. Steklov Inst. Math. 213, 104-114 (1996) Zbl 0932.32021 MR 1632237

[21] Kapovich, M.: Hyperbolic Manifolds and Discrete Groups. Progr. Math. 183, Birkhäuser Boston, Boston, MA (2001) Zbl 0958.57001 MR 1792613 
[22] Kobayashi, S.: Hyperbolic Manifolds and Holomorphic Mappings. An Introduction. 2nd ed., World Sci., Hackensack, NJ (2005) Zbl 1084.32018 MR 2194466

[23] Lehto, O., Virtanen, K. I.: Quasiconformal Mappings in the Plane. 2nd ed., Springer, Berlin (1973) Zbl 0267.30016 MR 0344463

[24] Lins Neto, A.: Algebraic solutions of polynomial differential equations and foliations in dimension two. In: Holomorphic Dynamics (Mexico, 1986), Lecture Notes in Math. 1345, Springer, 192-232 (1988) Zbl 0677.58036 MR 0980960

[25] Lins Neto, A.: Uniformization and the Poincaré metric on the leaves of a foliation by curves, Bol. Soc. Brasil. Mat. (N.S.) 31, 351-366 (2000) Zbl 0987.37039 MR 1817093

[26] Lins Neto, A., Pereira, J. V.: On the generic rank of the Baum-Bott map. Compos. Math. 142, 1549-1586 (2006) Zbl 1109.37041 MR 2278760

[27] Loray, F.: Sur les théorèmes I et II de Painlevé. In: Geometry and Dynamics, Contemp. Math. 389, Amer. Math. Soc., 165-190 (2005) Zbl 1148.34300 MR 2181964

[28] Loray, F., Rebelo, J.: Minimal, rigid foliations by curves on $\mathbb{P}^{n}$. J. Eur. Math. Soc. 5, 147-201 (2003) Zbl 1021.37030 MR 1985614

[29] Marín, D.: Problemas de módulos para una clase de foliaciones holomorfas. Tesis de Doctorado, Universitat Autònoma de Barcelona, 113-146 (2002); http://www.tdcat.cbuc.es/TDX0528101-120615/

[30] Painlevé, P.: Leçons sur la théorie analytique des équations différentielles professées à Stockholm. In: CEuvres Complètes, Tome I, Éditions du CNRS, Paris (1972)

[31] Palmeira, C. F. B.: Open manifolds foliated by planes. Ann. of Math. 107, 109-131 (1978) Zbl 0382.57010 MR 0501018

[32] de Saint-Gervais, H.-P.: Uniformisation des surfaces de Riemann. Retour sur un théorème centenaire. ENS Éditions, Lyon (2010) Zbl 1228.30001 MR 2768303

[33] Winkelnkemper, H. E.: The graph of a foliation, Ann. Global Anal. Geom. 1, 51-75 (1983) Zbl 0526.53039 MR 0739904 